\title{
Inhibitory Effect of Chemical Constituents Isolated from Artemisia iwayomogi on Polyol Pathway and Simultaneous Quantification of Major Bioactive Compounds
}

\author{
Yoon Kyoung Lee, Eun Young Hong, and Wan Kyunn Whang \\ Pharmaceutical Botany Laboratory, College of Pharmacy, Chung-Ang University, Heukseok-dong, Dongjak-gu, \\ Seoul 156-756, Republic of Korea
}

Correspondence should be addressed to Wan Kyunn Whang; whang-wk@cau.ac.kr

Received 28 February 2017; Revised 27 March 2017; Accepted 30 March 2017; Published 23 April 2017

Academic Editor: Sung-Hoon Kim

Copyright (c) 2017 Yoon Kyoung Lee et al. This is an open access article distributed under the Creative Commons Attribution License, which permits unrestricted use, distribution, and reproduction in any medium, provided the original work is properly cited.

Blocking the polyol pathway plays an important role preventing diabetic complications. Therefore, aldose reductase (AR) and advanced glycation endproducts (AGEs) formation has significant effect on diabetic complications. Artemisia iwayomogi has long been used as treatment of various diseases in Korea. However, no literatures have reported on AR and AGEs formation inhibitory activities of $A$. iwayomogi. For these reasons, we aimed to assess that $A$. iwayomogi had potential as anti-diabetic complications agents. We led to isolation of two coumarins (1 and 2), nine flavonoids (3-11), five caffeoylquinic acids (12-16), three diterpene glycosides (17-19), and one phenolic compound (20) from A. iwayomogi. Among them, hispidulin (4), 6-methoxytricin (6), arteanoflavone (7), quercetin-3-gentiobioside (10), 1,3-di-O-caffeoylquinic acid (13), and suavioside A (18) were first reported on the isolation from A. iwayomogi. Not only two coumarins (1 and $\mathbf{2}$ ), nine flavonoids (3-11), and five caffeoylquinic acids (12-16) but also extracts showed significant inhibitor on AR and AGEs formation activities. We analyzed contents of major bioactive compounds in Korea's various regions of A. iwayomogi. Overall, we selected Yangyang, Gangwon-do, from June, which contained the highest amounts of bioactive compounds, as suitable areas for cultivating A. iwayomogi as preventive or therapeutic agent in the treatment of diabetic complications.

\section{Introduction}

Aldose reductase (AR) belongs to the aldo-keto reductase family. It is the first and rate-controlling enzyme in the polyol pathway that reduces glucose to sorbitol using nicotinamide adenine dinucleotide $2^{\prime}$-phosphate (NADPH) as a cofactor $[1,2]$. Although blood sugar is high, the polyol pathway converts the excess glucose into sorbitol and then fructose. This accumulation of sorbitol and fructose has been demonstrated to be responsible for diabetic complications, including nephropathy, cataracts, neuropathy, and retinopathy [3, 4]. In particular, sorbitol is implicated in the pathogenesis of sugar cataracts, while the accumulation of fructose induces the formation of advanced glycation endproducts (AGEs), which are strongly implicated in diabetic complications and Alzheimer's disease [5]. Therefore, research on AR and AGEs formation inhibition is on the rise. There is a wide range of literature demonstrating that the developments of $\mathrm{AR}$ and AGEs formation are blocked by natural sources, especially plants that have an enormous content of bioactive compounds $[6,7]$.

Artemisia iwayomogi, locally called haninjin or dowijigi, is a member of the Compositae family and a perennial herb mainly found in Korea. A. iwayomogi has long been used in Korea in vegetables and foods such as tea, rice cake, and soup and also used for the treatment of various diseases including hepatitis, inflammation, and immune-related diseases as a protection for the liver, and a diuretic [8-10]. Previous studies have reported the isolation of scopoletin, esculetin 6methylether, scopolin, $\beta$-sitosterol, chlorogenic acid, quebrachitol, essential oils, fatty acids, sesquiterpene lactones, and flavonoids in A. iwayomogi [11-14]. Among them, scopolin modulated the expression of obesity-associated genes and was shown to have pharmacological effects on obesity, fatty 
liver, and diabetes [15]. A. iwayomogi has also demonstrated various biological activities. For example, methanol extracts of A. iwayomogi were shown to act as a scavenger of peroxynitrile, a compound involved in inducing or maintaining many diseases, including inflammation and aging [16]. Methanol extract of $A$. iwayomogi also inhibits nitric oxide in the production of lipopolysaccharide activated macrophages [17]. In other studies, a water-soluble carbohydrate fraction from A. iwayomogi suppressed spontaneous or 2,3, 7,8-tetrachlorodibenzo- $p$-dioxin-induced apoptotic death of mouse thymocytes by downregulating Fas gene expression $[18,19]$. A water-soluble carbohydrate fraction from A. iwayomogi also repressed pulmonary eosinophilia and Th2-type cytokine production in ovalbumin-induced allergic asthma via downregulation of TNF- $\alpha$ expression in the lungs [20], showed antitumor, immunomodulating activities [9], and modulated the functional differentiation of bone marrowderived dendritic cells [21].

However, A. iwayomogi's ability to inhibit AR and AGEs formation has never been previously studied. We investigated various components found in A. iwayomogi and their inhibitory activities on AR and AGEs formation. Our study also determined the bioactive compounds present in $A$. iwayomogi by region in Korea using an HPLC-PAD. Based on our study, we selected suitable areas for cultivating $A$. iwayomogi as medicinal foods.

\section{Materials and Methods}

2.1. Plant Materials. The aerial parts of A. iwayomogi were purchased from Kyung-Dong market, Seoul, Korea. Moreover, the aerial parts of A. iwayomogi were collected from Seoul, Gyeongii-do, Chungcheongbuk-do, Jeollabukdo, Jeollanam-do, Gyeongsangbuk-do, Gyeongsangnam-do, Gangwon-do, and Jeju-do Korea for analysis. Professor Whang Wan Kyunn identified A. iwayomogi which was bought in Kyung-Dong market and collected in various specimens.

2.2. Instruments and Reagents. Toluene, ethyl formate, chloroform $\left(\mathrm{CHCl}_{3}\right)$, ethyl acetate $(\mathrm{EtOAc})$, methanol $(\mathrm{MeOH})$, ethanol $(\mathrm{EtOH})$, distilled water, and formic acid were used as the open column chromatography and thin layer chromatography. Open column chromatography was used with Amberlite gel (Nippon Rensui Co., Japan), silica gel (200 400 mesh; Merck Co., Germany), Sephadex LH-20 (25-100 $\mu \mathrm{m}$; Pharmacia, Sweden), MCI CHP 20P (Supelco, USA), and ODS gel (400-500 mesh; Waters, USA). Thin layer chromatography was conducted with a Kiesel gel 60 $\mathrm{F}_{254}$ (Merck Co., Darmstadt, Germany) and plates (silica gel, $0.25 \mathrm{~mm}$ layer on aluminum sheets), and compounds were profiled by spraying with $10 \% \mathrm{H}_{2} \mathrm{SO}_{4}$ in $\mathrm{EtOH}$, followed by heating to $110^{\circ} \mathrm{C}$. Dimethyl sulfoxide- $d_{6}$ (DMSO- $\left.d_{6}\right)$, methanol- $d_{6}\left(\mathrm{CD}_{3} \mathrm{OD}\right)$, and pyridine- $d_{6}$ were used as the NMR solution. MS was performed using fast atom bombardment mass spectrometry (FAB-MS) conducted using a JEOL JMS-600W (Tokyo, Japan) mass spectrometer, a Q-TOF Synapt G2 apparatus (Waters, Milford, Massachusetts, USA) equipped with electrospray ionization (ESI), and electrospray ionization hybrid linear trap-quadruple-orbitrap (ESI/LTQOrbitrap) using an Ultimate 3000 RSLC (Thermo, Germany). ${ }^{1} \mathrm{H}$ and ${ }^{13} \mathrm{C}$-nuclear magnetic resonance (NMR) spectra were conducted at $600 \mathrm{MHz}$ and $150 \mathrm{MHz}$, respectively, with a Varian Gemini 2000 (USA) spectrometer using tetramethylsilane (TMS) as an internal standard. Chemical shifts are expressed as parts per million (ppm) on the $\delta$ scale, and coupling constants $(J)$ are showed in Hertz. HPLC was conducted using Empower Pro 2.0 software and determination was performed using a Waters 2695 system pump with a Waters 996 Photodiode array detector (USA). The separation column was a Waters Sunfire ${ }^{\mathrm{TM}}$ column C18 $(4.6 \times 250 \mathrm{~mm}, 5 \mu \mathrm{m})$. HPLC-grade solvents such as acetonitrile (ACN), methanol $(\mathrm{MeOH})$, and distilled water $\left(\mathrm{H}_{2} \mathrm{O}\right)$ were purchased from $\mathrm{J}$. T. Baker ${ }^{\circledR}$ (Phillipsburg, PA, USA). HPLC-grade formic acid and dimethyl sulfoxide (DMSO) were obtained from DEAJUNG Chemical (Siheung, Gyeonggi, Korea). Reagents and solvents including dl-glyceraldehyde, potassium phosphate buffer, nicotinamide adenine dinucleotide $2^{\prime}$-phosphate $(\beta$ NADPH), sodium phosphate buffer, sodium azide, 3,3tetramethyleneglutaric acid (TMG), bovine serum albumin, aminoguaidine (AG), glucose, and fructose were purchased from Sigma-Aldrich (St. Louis, MO, USA).

2.3. Extraction, Fractionation, and Isolation from A. iwayomogi. The dried and powdered aerial parts of A. iwayomogi $(5 \mathrm{~kg})$ were extracted with $50 \% \mathrm{EtOH}(20 \mathrm{~L} \times 3)$ at room temperature. The filtrate was concentrated to dryness (692.1 g) in vacuo, suspended in water $\left(\mathrm{H}_{2} \mathrm{O}\right)$, and then partitioned using $\mathrm{CHCl}_{3}(97.6 \mathrm{~g})$. After partitioning, the water fraction (456.7 g) was chromatographed on a Amberlite XAD-2 using water, 30\% methanol, $60 \%$ methanol, and $100 \%$ methanol. The result yielded water ( $240 \mathrm{~g}), 30 \%$ methanol ( $92.18 \mathrm{~g}), 60 \%$ methanol (49.31 g), and 100\% methanol (70.24 g) fractions.

The $\mathrm{CHCl}_{3}$ fraction $(97.6 \mathrm{~g}$ ) was chromatographed with a silica gel solvent system to increase the elution power of $\mathrm{CHCl}_{3}: \mathrm{MeOH}(20: 1$ to $0: 100)$ and yielded ten subfractions. Subfraction 2 (25.62 g) was separated using Sephadex LH-20 column chromatography with $50 \% \mathrm{MeOH}$ to obtain fractions 2-1 2-11. Compound 1 (1.16 g) was isolated from fraction 2-2. In addition, separation of subfraction 2-3 (6.39g) using an MCI gel with $50 \% \mathrm{MeOH}$ solvent system yielded fourteen fractions. Subfraction 2-3-10 led to isolation of compound 3 (34.4 mg). Fraction 2-3-6 (632.5 mg) was separated using ODS column chromatography with a $60 \% \mathrm{MeOH}$ elution solvent system to collect fractions 2-3-6-1 2-3-6-4. Compound 5 (97.5 $\mathrm{mg}$ ) was isolated from subfraction $2-3-6-2$ by recrystallization, and then a portion of subfraction 2-3-6-2 (324.2 mg) was used on a Sephadex LH-20 (50\% MeOH), to yield compound 6 (13.2 mg). Subfraction 2-3-9 (448.4 $\mathrm{mg})$ was separated using ODS $(60 \% \mathrm{MeOH}), \mathrm{MCI}(50 \%$ $\mathrm{MeOH})$, and Sephadex LH-20 (40\% EtOH), which yielded compound $7(17.5 \mathrm{mg})$. Fraction $3(2.38 \mathrm{~g})$ was applied to a Sephadex LH-20 column chromatography with a gradient solvent system of $50 \%$ to $100 \% \mathrm{MeOH}$ and yielded fourteen fractions. Fraction 3-9 (96.8 mg) was separated using ODS column chromatography with $70 \% \mathrm{MeOH}$, which obtained 
six fractions (3-9-1 3-9-6). Subfraction 3-9-3 (20 mg) was chromatographed on a Sephadex LH-20 using $50 \% \mathrm{MeOH}$ to yield compound 4 (19.9 mg).

A portion of the $100 \% \mathrm{MeOH}$ fraction $(15 \mathrm{~g})$ was separated using a Sephadex LH-20 column chromatography with a gradient elution solvent system of $20 \%$ to $100 \% \mathrm{MeOH}$ to give eighteen subfractions. Subfraction $3(0.93 \mathrm{~g})$ was applied to ODS column chromatography using a $10 \% \mathrm{MeOH}$ solvent system to yield compound $\mathbf{1 2}(37.9 \mathrm{mg})$. Subfraction $16(0.99 \mathrm{~g})$ was separated using a ODS column chromatography with $20 \% \mathrm{MeOH}$, and ten fractions (16-1 16-10) were collected. Fraction 16-6 (134.3 mg) led to the isolation of compound $14(70.0 \mathrm{mg})$ by recrystallization. Fraction 16-5 (395.7 mg) was separated by a Sephadex LH-20 using an eluent of $40 \% \mathrm{EtOH}$, which yielded compounds $15(272.2 \mathrm{mg})$ and $\mathbf{1 6}(72.2 \mathrm{mg})$.

The $60 \% \mathrm{MeOH}$ fraction (49.31 g) was separated using a Sephadex LH-20 column chromatography with a gradient of $40 \% \mathrm{MeOH}$ to $100 \% \mathrm{MeOH}$, which yielded twelve subfractions. Subfraction 1 (6.7 g) was separated by an MCI gel using a $50 \% \mathrm{MeOH}$ to $100 \% \mathrm{MeOH}$ solvent system, which yielded fifteen fractions (1-1 1-15). Fractions 1-8 (331.6 mg), 1-9 $(101.6 \mathrm{mg})$, and 1-10 $(157.5 \mathrm{mg})$ were isolated by recrystallization to yield compounds $17(32.7 \mathrm{mg}), \mathbf{1 8}(9.2 \mathrm{mg})$, and 19 (28.6 mg), respectively. Subfraction $3(923.4 \mathrm{mg})$ was separated using an ODS column chromatography, and seven fractions (3-1 3-7) were collected after elution with $40 \%$ $\mathrm{MeOH}$. Fraction 3-3 (140.4 mg) was separated by MCI column chromatography using an eluent of $50 \% \mathrm{MeOH}$, leading to the isolation of compound 20. Subfraction $5(1.48 \mathrm{~g})$ was separated using an MCI gel and eluted with $50 \% \mathrm{MeOH}$, which yielded compound $\mathbf{8}(281.7 \mathrm{mg})$. A portion of fraction 5-3 (166.2 mg) was applied to an MCI gel and eluted with $40 \% \mathrm{MeOH}$. Fraction 5-3-3 (106 mg) was separated by a Sephadex LH-20 column chromatography using an eluent of $50 \% \mathrm{EtOH}$ to yield compounds $\mathbf{1 0}(23.1 \mathrm{mg})$ and $\mathbf{1 1}(36.8 \mathrm{mg})$. Subfraction $7(806.2 \mathrm{mg})$ and subfraction $8(3.77 \mathrm{~g})$ were chromatographed on an MCI gel using $40 \% \mathrm{MeOH}$, to give fractions 7-1 7-10 and 8-1 8-12, respectively. Compound 9 (76.6 mg) was isolated from fraction 7-4 (108.8 mg) using a Sephadex LH-20 eluting with 50\% EtOH. Subfraction 8-2 ( $3.77 \mathrm{~g})$ was separated using a Sephadex LH-20 eluting with $40 \% \mathrm{MeOH}$. Fraction 8-2-3 was applied to a Sephadex LH20 column chromatography using $20 \% \mathrm{EtOH}$, which yielded compound $\mathbf{1 3}$ (111.1 mg).

A portion of the $30 \% \mathrm{MeOH}$ fraction $(14 \mathrm{~g})$ was chromatographed on a Sephadex LH-20 column chromatography using a solvent system of $20 \% \mathrm{MeOH}$ to $100 \% \mathrm{MeOH}$, which yielded seven subfractions. Fraction $2(7.12 \mathrm{mg})$ was separated using an ODS column chromatography, and fifteen fractions (2-1 2-15) were collected after elution with 5\% $\mathrm{MeOH}$. Subfraction 2-6 was applied to an MCI gel using 5\% $\mathrm{EtOH}$, which yielded compound 2 (171.4 mg).

2.4. Measurement of AR Inhibitory Activity. Rat lenses (one lens per $0.5 \mathrm{~mL}$ sodium buffer) were obtained from SpragueDawley rats (weighing 250 280 g). The rat lenses were homogenized in $0.1 \mathrm{M}$ sodium phosphate buffer ( $\mathrm{pH} 6.2$ ) and centrifuged at $10,000 \mathrm{rpm}\left(4^{\circ} \mathrm{C}, 20 \mathrm{~min}\right)$. After centrifugation, the supernatant was used as an enzyme. AR activity was spectrophotometrically determined by measuring the decrease in the absorption of $\beta-\mathrm{NADPH}$ at $340 \mathrm{~nm}$ for a 4 min period using DL-glyceraldehydes as substrates [22]. The assay mixture contained $1.6 \mathrm{mM} \beta$-NADPH, $0.1 \mathrm{M}$ potassium phosphate buffer ( $\mathrm{pH}$ 7.0), AR homogenate, $4 \mathrm{M}$ ammonium sulfate, $0.025 \mathrm{M}$ DL-glyceraldehyde, and the sample in $100 \%$ DMSO. The total volume of the assay mixture was $0.3 \mathrm{~mL}$ and the reaction was performed in a 96-well plate. 3,3'Tetramethylene glutaric acid (TMG), a typical AR inhibitor, was used as a positive control.

2.5. Measurement of AGEs Formation Inhibitory Activity. Inhibition of AGEs formation was determined by an assay containing bovine serum albumin $(10 \mathrm{mg} / \mathrm{mL})$ in $50 \mathrm{mM}$ phosphate buffer ( $\mathrm{pH} 7.4$ ) with $0.02 \%$ sodium azide, to which $0.4 \mathrm{M}$ fructose and glucose was added. The assay mixture was incubated at $60^{\circ} \mathrm{C}$ for 2 days. After incubating, the reaction was measured on Fluorescence in a 96-black-well plate (excitation wavelength $350 \mathrm{~nm}$, emission wavelength $450 \mathrm{~nm}$ ) [23]. Aminoguaidine (AG) was used as a positive control.

2.6. For $A R$ and AGEs Formation Inhibition Assays, the Activity Was Calculated. Inhibition (\%): (Ac-As/Ac) $\times 100$. In AR inhibition activity, Ac is absorbance of control and As is absorbance of samples. In AGEs formation inhibition activity, Ac is fluorescence of control and As is fluorescence of samples. $\mathrm{IC}_{50}$ is the concentration of inhibitor that gives a $50 \%$ inhibition in enzyme activity. $\mathrm{IC}_{50}$ values were calculated from the least-squares regression line of the log of concentration plotted against residual activity. All assays were performed in triplicate. Data was presented as mean \pm standard deviation (SD).

2.7. Sample Preparation for HPLC. Samples of A. iwayomogi taken from the various regions in Korea and Yangyang, Gangwon-do, monthly between June and October were dissolved in $50 \% \mathrm{EtOH}(20 \mathrm{mg} / \mathrm{mL})$. The resulting solution was filtered with a $0.45 \mu \mathrm{m}$ syringe filter. The resulting solution was used for HPLC analysis.

2.8. HPLC Conditions. A Waters Sunfire ${ }^{\mathrm{TM}}$ column $\mathrm{C} 18$ ( $4.6 \times$ $250 \mathrm{~mm}, 5 \mu \mathrm{m}$ ) was used for the determination of compounds $1,2,8,10,11,12,14$, and 15 . The mobile phase consisted of $0.1 \%$ formic acid (solvent A) and ACN (solvent B). The gradient solvent system was solvents A and B $(85: 15)$ and increased in linear gradients to $84: 16$ for $5 \mathrm{~min}$, to $72: 28$ for $5 \mathrm{~min}$, then to $69: 31$ for $5 \mathrm{~min}$, and finally to $40: 60$ for $5 \mathrm{~min}$. The injection volume was $10 \mu \mathrm{L}$ and the flow rate was $0.9 \mathrm{~mL} / \mathrm{min}$. The UV spectra were recorded at $330 \mathrm{~nm}$ for quantification of compounds. All injections were performed in triplicate.

2.9. Calibration Curve. Stock solutions of compounds $\mathbf{1}, \mathbf{2}, \mathbf{8}$, $10,11,12,14$, and 15 in 50\% EtOH were prepared in several concentrations. Calibration curves of the eight standards were calculated using concentration $(X, \mu \mathrm{g} / \mathrm{mL})$, peak area 
$(Y)$, and mean value $(n=3) \pm$ standard deviation. Contents of the analyte solutions were then determined from the calibration curves.

2.10. Determination of Limit of Detection (LOD) and Limit of Quantification (LOQ). Quantification of the HPLC method for compounds $1,2,8,10,11,12,14$, and 15 as a standard compound was determined by LOD and LOQ. The LOD and LOQ were defined as detectable concentration of the compound with a signal to noise $(S / N)$ ratio of $\geq 3.3$ and $\geq 10$, respectively. The percent recovery was evaluated by calculating the ratio of the amount detected versus the amount added. The quantity of analysis was subsequently obtained from the calibration curve.

\section{Results}

3.1. Identification of Compounds 1-20 Isolated from A. iwayomogi. Chromatographic separation of the $50 \% \mathrm{EtOH}$ extraction from $A$. iwayomogi led to the isolation of two coumarins (1 and 2), nine flavonoids (3-11), five caffeoylquinic acids (12-16), three diterpene glycosides (17-19), and one phenolic compound (20) (Figure 1). Compounds 1-20 isolated from $A$. iwayomogi were identified as scopoletin (1) [11, 24], scopolin (2) [11, 24], genkwanin (3) [11], hispidulin (4) [25], jaceosidin (5) [11, 26], 6methoxytricin (6) [26], arteanoflavone (7) [27], patuletin3-O-glucoside (8) [28], isoquercetin (9) [24, 29], quercetin3-gentiobioside (10) [29], rutin (11) [30], 3-O-caffeoylquinic acid (12) [24], 1,3-di-O-caffeoylquinic acid (13) [31], 3,4-di$O$-caffeoylquinic acid (14) [32], 3,5-di- $O$-caffeoylquinic acid (15) $[24,32]$, 3,5-di-O-caffeoylquinic acid methyl ester (16) [32], iwayoside A (17) [33], suavioside A (18) [34], sugeroside (19) [34], and 2,4-dihydroxy-6-methoxyacetophenone 4-O- $\beta$-D-glucopyranoside (20) [11] by comparison with the spectroscopic data $\left({ }^{1} \mathrm{H}-\mathrm{NMR},{ }^{13} \mathrm{C}-\mathrm{NMR}\right.$, and MS) from the literature.

Compound 1: $\mathrm{C}_{10} \mathrm{H}_{8} \mathrm{O}_{4}$; Q-TOF-MS $m / z: 193.0490$ $[\mathrm{M}+\mathrm{H}]^{+} ;{ }^{1} \mathrm{H}-\mathrm{NMR}\left(600 \mathrm{MHz}, \mathrm{DMSO}-d_{6}\right) \delta: 7.93(1 \mathrm{H}, \mathrm{d}, J=$ $9.6 \mathrm{~Hz}, \mathrm{H}-4), 7.24$ (1H, s, H-5), 6.81 (1H, s, H-8), 6.25 (1H, 3, $J=9.0 \mathrm{~Hz}, \mathrm{H}-3), 3.85(3 \mathrm{H}, \mathrm{s}, 6-\mathrm{OMe}) ;{ }^{13} \mathrm{C}-\mathrm{NMR}(150 \mathrm{MHz}$, DMSO- $\left.d_{6}\right) \delta: 162.5$ (C-2), 152.8 (C-7), 151.3 (C-9), 147.0 (C-6), 146.3 (C-4), 113.5 (C-3, 10), 112.4 (C-5), 104.5 (C-8), 57.8 (6-OMe).

Compound 2: $\mathrm{C}_{16} \mathrm{H}_{18} \mathrm{O}_{9}$; Q-TOF-MS m/z: 353.0869 $[\mathrm{M}-\mathrm{H}]^{-} ;{ }^{1} \mathrm{H}-\mathrm{NMR}\left(600 \mathrm{MHz}, \mathrm{DMSO}-d_{6}\right): 8.00(1 \mathrm{H}, \mathrm{d}, J=$ $9.0 \mathrm{~Hz}, \mathrm{H}-4), 7.33(1 \mathrm{H}, \mathrm{d}, J=1.8 \mathrm{~Hz}, \mathrm{H}-5), 7.19(1 \mathrm{H}, \mathrm{s}, \mathrm{H}-8)$, $6.36(1 \mathrm{H}, 3, J=9.0 \mathrm{~Hz}, \mathrm{H}-3), 5.12(1 \mathrm{H}, \mathrm{d}, J=7.2 \mathrm{~Hz}$, Glc H-1), $3.86(3 \mathrm{H}, \mathrm{s}, 6-\mathrm{OMe}) ;{ }^{13} \mathrm{C}-\mathrm{NMR}\left(150 \mathrm{MHz}, \mathrm{DMSO}-d_{6}\right): 161.0$ (C-2), 150.2 (C-7), 147.2 (C-9), 146.3 (C-6), 144.6 (C-4), 113.7 (C-3), 112.7 (C-10), 110.0 (C-5), 103.4 (C-8), 100.0 (Glc C-1), 77.4 (Glc C-3), 76.9 (Glc C-5), 73.3 (Glc C-2), 69.9 (Glc C-4), 60.9 (Glc C-6), 56.4 (6-OMe).

Compound 3: $\mathrm{C}_{16} \mathrm{H}_{12} \mathrm{O}_{5}$; ESI/LTQ-Orbitrap-MS $\mathrm{m} / z$ : $285.08[\mathrm{M}+\mathrm{H}]^{+} ;{ }^{1} \mathrm{H}-\mathrm{NMR}\left(600 \mathrm{MHz}, \mathrm{DMSO}-d_{6}\right): 12.93(5-$ $\mathrm{OH}), 7.96\left(2 \mathrm{H}, \mathrm{d}, J=9.0 \mathrm{~Hz}, \mathrm{H}-2^{\prime}, 6^{\prime}\right), 6.94(2 \mathrm{H}, \mathrm{d}, J=9.0 \mathrm{~Hz}$, H-3', $\left.5^{\prime}\right), 6.84(1 \mathrm{H}, \mathrm{s}, \mathrm{H}-3), 6.77$ (1H, s, H-8), 6.37 (1H, s, H-6), 3.87 (3H, s, 7-OMe); ${ }^{13} \mathrm{C}-\mathrm{NMR}\left(150 \mathrm{MHz}, \mathrm{DMSO}-d_{6}\right): 182.3$
(C-4), 165.5 (C-7), 164.5 (C-2), 161.8 (C-4' $), 161.6$ (C-9), 157.6 $(\mathrm{C}-5), 133.5(\mathrm{C}-3), 128.9\left(\mathrm{C}-2^{\prime}, 6^{\prime}\right), 121.4\left(\mathrm{C}-1^{\prime}\right), 116.4\left(\mathrm{C}-3^{\prime}, 5^{\prime}\right)$, 103.9 (C-10), 98.7 (C-6), 93.5 (C-8), 56.5 (7-OMe).

Compound 4: $\mathrm{C}_{16} \mathrm{H}_{12} \mathrm{O}_{6}$; ESI/LTQ-Orbitrap-MS $\mathrm{m} / z$ : $301.07[\mathrm{M}+\mathrm{H}]^{+} ;{ }^{1} \mathrm{H}-\mathrm{NMR}\left(600 \mathrm{MHz}, \mathrm{DMSO}-d_{6}\right): 13.04$ (5$\mathrm{OH}), 7.88\left(2 \mathrm{H}, \mathrm{d}, J=8.4 \mathrm{~Hz}, \mathrm{H}-2^{\prime}, 6^{\prime}\right), 6.90(2 \mathrm{H}, \mathrm{d}, J=8.4 \mathrm{~Hz}$, H-3', $\left.5^{\prime}\right), 6.72(1 \mathrm{H}, \mathrm{s}, \mathrm{H}-3), 6.55(1 \mathrm{H}, \mathrm{s}, \mathrm{H}-8), 3.73(3 \mathrm{H}, \mathrm{s}, 6-$ $\mathrm{OMe}) ;{ }^{13} \mathrm{C}-\mathrm{NMR}\left(150 \mathrm{MHz}, \mathrm{DMSO}-d_{6}\right): 182.3$ (C-4), 164.0 (C-2), 161.4 (C-4'), 157.8 (C-7), 153.1 (C-5), 152.7 (C-9), 131.7 (C-6), $128.7\left(\mathrm{C}-2^{\prime}, 6^{\prime}\right), 121.5\left(\mathrm{C}-1^{\prime}\right), 116.2\left(\mathrm{C}-3^{\prime}, 5^{\prime}\right), 104.3(\mathrm{C}-3)$, 102.7 (C-10), 94.6 (C-8), 60.2 (6-OMe).

Compound 5: $\mathrm{C}_{17} \mathrm{H}_{14} \mathrm{O}_{7}$; ESI/LTQ-Orbitrap-MS $\mathrm{m} / z$ : $331.08[\mathrm{M}+\mathrm{H}]^{+} ;{ }^{1} \mathrm{H}-\mathrm{NMR}\left(600 \mathrm{MHz}, \mathrm{DMSO}-d_{6}\right): 13.06(5-$ $\mathrm{OH}), 7.53\left(2 \mathrm{H}, \mathrm{s}, \mathrm{H}-5^{\prime}, 6^{\prime}\right), 6.91\left(1 \mathrm{H}, \mathrm{d}, J=9.0 \mathrm{~Hz}, \mathrm{H}-2^{\prime}\right), 6.86$ $(1 \mathrm{H}, \mathrm{s}, \mathrm{H}-3), 6.58(1 \mathrm{H}, \mathrm{s}, \mathrm{H}-8), 3.88(3 \mathrm{H}, \mathrm{s}, 6-\mathrm{OMe}), 3.74(3 \mathrm{H}, \mathrm{s}$, $3^{\prime}$-OMe); ${ }^{13} \mathrm{C}-\mathrm{NMR}\left(150 \mathrm{MHz}, \mathrm{DMSO}-d_{6}\right): 182.5$ (C-4), 164.1 (C-2), 158.1 (C-7), 153.1 (C-9), 152.9 (C-5), 151.2 (C-4'), 148.4 $\left(\mathrm{C}-3^{\prime}\right), 131.9(\mathrm{C}-6), 121.9\left(\mathrm{C}-1^{\prime}\right), 120.7\left(\mathrm{C}-6^{\prime}\right), 116.2\left(\mathrm{C}-5^{\prime}\right), 110.6$ (C-2' $), 104.3$ (C-10), 103.1 (C-3), 94.8 (C-8), 60.3 (6-OMe), $56.4\left(3^{\prime}\right.$-OMe).

Compound 6: $\mathrm{C}_{18} \mathrm{H}_{16} \mathrm{O}_{8} ;$ ESI/LTQ-Orbitrap-MS $\mathrm{m} / z$ : $361.09[\mathrm{M}+\mathrm{H}]^{+} ;{ }^{1} \mathrm{H}-\mathrm{NMR}\left(600 \mathrm{MHz}, \mathrm{DMSO}-d_{6}\right): 13.04(5-$ $\mathrm{OH}), 7.29\left(2 \mathrm{H}, \mathrm{s}, \mathrm{H}-2^{\prime}, 6^{\prime}\right), 6.93(1 \mathrm{H}, \mathrm{s}, \mathrm{H}-3), 6.64(1 \mathrm{H}, \mathrm{s}, \mathrm{H}-$ 8), 3.86 (3H, s, 6-OMe), 3.73 (6H, s, $3^{\prime}, 5^{\prime}$-OMe); ${ }^{13} \mathrm{C}-\mathrm{NMR}$ $\left(150 \mathrm{MHz}, \mathrm{DMSO}-d_{6}\right)$ : 182.5 (C-4), 164.0 (C-2), 158.1 (C-7), $153.1(\mathrm{C}-5), 152.9$ (C-9), $148.6\left(\mathrm{C}-3^{\prime}, 5^{\prime}\right), 140.3\left(\mathrm{C}-4^{\prime}\right), 131.9(\mathrm{C}-$

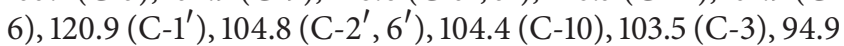
(C-8), 60.3 (6-OMe), 56.8 (3', $5^{\prime}$-OMe).

Compound 7: $\mathrm{C}_{18} \mathrm{H}_{18} \mathrm{O}_{8}$; ESI/LTQ-Orbitrap-MS $\mathrm{m} / z$ : $375.11[\mathrm{M}+\mathrm{H}]^{+} ;{ }^{1} \mathrm{H}-\mathrm{NMR}\left(600 \mathrm{MHz}, \mathrm{DMSO}-d_{6}\right):{ }^{1} \mathrm{H}-\mathrm{NMR}$ $\left(600 \mathrm{MHz}, \mathrm{DMSO}-d_{6}\right): 12.91(5-\mathrm{OH}), 7.28\left(2 \mathrm{H}, \mathrm{s}, \mathrm{H}-2^{\prime}, 6^{\prime}\right)$, $6.99(1 \mathrm{H}, \mathrm{s}, \mathrm{H}-3), 6.63(1 \mathrm{H}, \mathrm{s}, \mathrm{H}-8), 3.87\left(6 \mathrm{H}, \mathrm{s}, 6,4^{\prime}-\mathrm{OMe}\right)$, $3.73\left(6 \mathrm{H}, \mathrm{s}, 3^{\prime}, 5^{\prime}-\mathrm{OMe}\right) ;{ }^{13} \mathrm{C}-\mathrm{NMR}\left(150 \mathrm{MHz}, \mathrm{DMSO}-d_{6}\right)$ : 182.5 (C-4), 163.4 (C-2), 158.7 (C-7), 153.7 (C-2', $\left.5^{\prime}\right), 153.1$ (C5), 152.5 (C-9), $141.1\left(\mathrm{C}-4^{\prime}\right), 132.1(\mathrm{C}-6), 126.5\left(\mathrm{C}-1^{\prime}\right), 104.9(\mathrm{C}-$ $\left.2^{\prime}\right), 104.5\left(\mathrm{C}-6^{\prime}\right), 104.2(\mathrm{C}-10), 103.8(\mathrm{C}-3), 95.2(\mathrm{C}-8), 60.6$ (6$\mathrm{OMe}), 60.3$ ( $4^{\prime}$-OMe), $56.7\left(3^{\prime}, 5^{\prime}\right.$-OMe).

Compound 8: $\mathrm{C}_{22} \mathrm{H}_{22} \mathrm{O}_{13}$; FAB-MS m/z: $495[\mathrm{M}+\mathrm{H}]^{+}$; ${ }^{1} \mathrm{H}-\mathrm{NMR}\left(600 \mathrm{MHz}, \mathrm{DMSO}-d_{6}\right): 13.01(5-\mathrm{OH}), 7.69(1 \mathrm{H}, \mathrm{d}, J$ $\left.=2.4 \mathrm{~Hz}, \mathrm{H}-2^{\prime}\right), 7.58\left(1 \mathrm{H}, \mathrm{dd}, J=2.4,8.4 \mathrm{~Hz}, \mathrm{H}-6^{\prime}\right), 6.86(1 \mathrm{H}, \mathrm{d}$, $\left.J=8.4 \mathrm{~Hz}, \mathrm{H}-5^{\prime}\right), 6.48(1 \mathrm{H}, \mathrm{s}, \mathrm{H}-8), 5.26(1 \mathrm{H}, \mathrm{d}, J=7.8 \mathrm{~Hz}, \mathrm{Glc}$ $\mathrm{H}-1), 3.86$ (3H, s, 6-OMe); ${ }^{13} \mathrm{C}-\mathrm{NMR}$ (150 MHz, DMSO- $\left.d_{6}\right)$ : 179.4 (C-4), 158.8 (C-9), 158.5 (C-2), 153.5 (C-5), 153.3 (C-7), $149.5\left(\mathrm{C}-4^{\prime}\right), 145.6\left({\mathrm{C}-5^{\prime}}^{\prime}\right), 135.0(\mathrm{C}-3), 132.3$ (C-6), 122.9 (C$\left.1^{\prime}\right), 122.8\left(\mathrm{C}-6^{\prime}\right), 117.2\left(\mathrm{C}-2^{\prime}\right), 115.7\left(\mathrm{C}-3^{\prime}\right), 105.8(\mathrm{C}-10), 103.9$ (Glc C-1), 94.6 (C-8), 78.1 (Glc C-5), 77.8 (Glc C-3), 75.4 (Glc C-2), 70.9 (Glc C-4), 62.2 (Glc C-6), 60.6 (6-OMe).

Compound 9: $\mathrm{C}_{22} \mathrm{H}_{20} \mathrm{O}_{12}$; FAB-MS m/z: $465[\mathrm{M}+\mathrm{H}]^{+}$; ${ }^{1} \mathrm{H}-\mathrm{NMR}\left(600 \mathrm{MHz}, \mathrm{DMSO}-d_{6}\right): 13.01(5-\mathrm{OH}), 7.55(1 \mathrm{H}, \mathrm{d}, J$ $\left.=1.2 \mathrm{~Hz}, \mathrm{H}-2^{\prime}\right), 7.54\left(1 \mathrm{H}, \mathrm{dd}, J=1.2,9.0 \mathrm{~Hz}, \mathrm{H}-6^{\prime}\right), 6.84(1 \mathrm{H}, \mathrm{d}$, $\left.J=9.0 \mathrm{~Hz}, \mathrm{H}-5^{\prime}\right), 6.36(1 \mathrm{H}, \mathrm{s}, \mathrm{H}-8), 6.15(1 \mathrm{H}, \mathrm{s}, \mathrm{H}-6), 5.41(1 \mathrm{H}$, d, $J=7.8 \mathrm{~Hz}$, Glc H-1); ${ }^{13} \mathrm{C}-\mathrm{NMR}\left(150 \mathrm{MHz}, \mathrm{DMSO}-d_{6}\right): 177.4$ (C-4), 161.1 (C-7), 157.2 (C-5), 156.7 (C-2), 156.3 (C-9), 148.6 $\left(\mathrm{C}-4^{\prime}\right), 144.9\left(\mathrm{C}-3^{\prime}\right), 133.5(\mathrm{C}-3), 121.9\left(\mathrm{C}-6^{\prime}\right), 121.4\left(\mathrm{C}-1^{\prime}\right), 116.3$ $\left(\mathrm{C}-5^{\prime}\right), 115.4\left(\mathrm{C}-2^{\prime}\right), 103.7$ (C-10), 101.2 (Glc C-1), 99.2 (C-6), 94.6 (C-8), 77.6 (Glc C-5), 76.5 (Glc C-3), 74.2 (Glc C-2), 70.1 (Glc C-4), 61.0 (Glc C-6).

Compound 10: $\mathrm{C}_{27} \mathrm{H}_{30} \mathrm{O}_{17}$; ESI/LTQ-Orbitrap-MS $m / z$ : $649.45[\mathrm{M}+\mathrm{Na}]^{+} ;{ }^{1} \mathrm{H}-\mathrm{NMR}\left(600 \mathrm{MHz}, \mathrm{DMSO}-d_{6}\right):{ }^{1} \mathrm{H}-\mathrm{NMR}$ 
Genkwanin (3)

Hispidulin (4)

Jaceosidin (5)

6-Methoxytricin (6)

Arteanoflavone (7)

Patuletin-3-O-glucoside (8)

Isoquercetin (9)

Quercetin-3-gentiobioside (10)

Rutin (11)<smiles></smiles>

$\begin{array}{cccccc}\mathrm{R}_{1} & \mathrm{R}_{2} & \mathrm{R}_{3} & \mathrm{R}_{4} & \mathrm{R}_{5} & \mathrm{R}_{6} \\ \mathrm{Me} & \mathrm{H} & \mathrm{H} & \mathrm{H} & \mathrm{H} & \mathrm{H} \\ \mathrm{H} & \mathrm{OMe} & \mathrm{H} & \mathrm{H} & \mathrm{H} & \mathrm{H} \\ \mathrm{H} & \mathrm{OMe} & \mathrm{H} & \mathrm{H} & \mathrm{H} & \mathrm{OMe} \\ \mathrm{H} & \mathrm{OMe} & \mathrm{H} & \mathrm{H} & \mathrm{OMe} & \mathrm{OMe} \\ \mathrm{H} & \mathrm{OMe} & \mathrm{H} & \mathrm{Me} & \mathrm{OMe} & \mathrm{OMe} \\ \mathrm{H} & \mathrm{OMe} & \text { O-Glucose } & \mathrm{H} & \mathrm{OH} & \mathrm{H} \\ \mathrm{H} & \mathrm{H} & \text { O-Glucose } & \mathrm{H} & \mathrm{OH} & \mathrm{H} \\ \mathrm{H} & \mathrm{H} & \text { O-Glucose-glucose } & \mathrm{H} & \mathrm{OH} & \mathrm{H} \\ \mathrm{H} & \mathrm{H} & \text { O-Glucose-rhamnose } & \mathrm{H} & \mathrm{OH} & \mathrm{H}\end{array}$<smiles>[R6]OC1CC([R6])(C([R6])O)CC([R6])C1O[R20]</smiles><smiles>CC(=O)/C=C/c1ccc(O)c(O)c1</smiles>

Caffeoyl $=$

3-O-Caffeoylquinic acid (12)

1,3-Di-O-caffeoylquinic acid (13)

3,4-Di-O-caffeoylquinic acid (14)

3,5-Di-O-caffeoylquinic acid (15)

3,5-Di-O-caffeoylquinic acid methyl ester (16)

$\begin{array}{ccccc}\mathrm{R}_{1} & \mathrm{R}_{2} & \mathrm{R}_{3} & \mathrm{R}_{4} & \mathrm{R}_{5} \\ \text { Caffeoyl } & \mathrm{H} & \mathrm{H} & \mathrm{H} & \mathrm{H} \\ \text { Caffeoyl } & \mathrm{H} & \mathrm{H} & \text { Caffeoyl } & \mathrm{H} \\ \text { Caffeoyl } & \text { Caffeoyl } & \mathrm{H} & \mathrm{H} & \mathrm{H} \\ \text { Caffeoyl } & \mathrm{H} & \text { Caffeoyl } & \mathrm{H} & \mathrm{H} \\ \text { Caffeoyl } & \mathrm{H} & \text { Caffeoyl } & \mathrm{H} & \mathrm{Me}\end{array}$<smiles>[R]C1CCC2(C)C3CCC4CC3(CCC2C1(C)C)CC4(O)COC1OC(CO)C(O)C(O)C1O</smiles>

$\mathrm{R}$
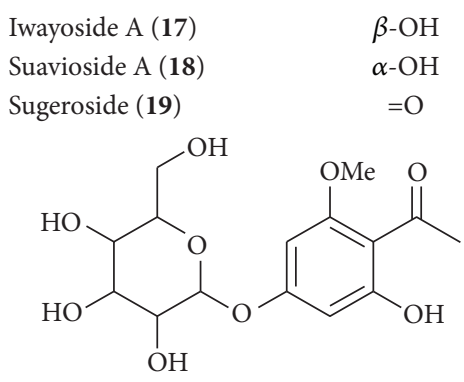

2,4-Dihydroxy-6-methoxyacetophenone 4-O- $\beta$-D-glucopyranoside (20)

FIGURE 1: Structures of the compounds 1-20. 
$\left(600 \mathrm{MHz}, \mathrm{DMSO}-d_{6}\right): 13.04(5-\mathrm{OH}), 7.64(1 \mathrm{H}, \mathrm{d}, J=2.4 \mathrm{~Hz}$, $\left.\mathrm{H}-2^{\prime}\right), 7.51\left(1 \mathrm{H}, \mathrm{dd}, J=2.4,8.4 \mathrm{~Hz}, \mathrm{H}-6^{\prime}\right), 6.82(1 \mathrm{H}, \mathrm{d}, J=$ $\left.8.4 \mathrm{~Hz}, \mathrm{H}-5^{\prime}\right), 6.36$ (1H, s, H-8), 6.14 (1H, s, H-6), 5.31 (1H, d, J $=7.8 \mathrm{~Hz}$, Glc H-1), $5.29\left(1 \mathrm{H}, \mathrm{d}, J=7.8 \mathrm{~Hz}\right.$, Glc H-1 $\left.{ }^{\prime}\right) ;{ }^{13} \mathrm{C}-\mathrm{NMR}$ $\left(150 \mathrm{MHz}, \mathrm{DMSO}-d_{6}\right)$ : 177.4 (C-4), 165.3 (C-5), 161.2 (C-7), 156.7 (C-2), 155.8 (C-9), $148.6\left(\mathrm{C}-3^{\prime}\right), 144.9\left(\mathrm{C}-4^{\prime}\right), 133.3(\mathrm{C}-$ 3), $122.3\left(\mathrm{C}-6^{\prime}\right), 121.5\left(\mathrm{C}-1^{\prime}\right), 115.9\left(\mathrm{C}-5^{\prime}\right), 115.4\left(\mathrm{C}-2^{\prime}\right), 108.9$ (C-10), 103.8 (Glc C-1), 99.2 (Glc C-1' ), 98.8 (C-6), 94.0 (C-8), 79.3 (Glc C-5), 77.6 (Glc C-3), 77.5 (Glc C-5'), 77.1 (Glc C-3'), 76.4 (Glc C-2, 2' ), 74.2 (Glc C-4), 70.3 (Glc C-4'), 64.3 (Glc C-6), 60.9 (Glc C-6').

Compound 11: $\mathrm{C}_{27} \mathrm{H}_{30} \mathrm{O}_{16}$; FAB-MS m/z: $611[\mathrm{M}+\mathrm{H}]^{+}$; ${ }^{1} \mathrm{H}-\mathrm{NMR}\left(600 \mathrm{MHz}, \mathrm{DMSO}-d_{6}\right): 13.01(5-\mathrm{OH}), 7.53(1 \mathrm{H}, \mathrm{d}, J$ $\left.=2.4 \mathrm{~Hz}, \mathrm{H}-2^{\prime}\right), 7.51\left(1 \mathrm{H}, \mathrm{dd}, J=2.4,8.4 \mathrm{~Hz}, \mathrm{H}-6^{\prime}\right), 6.83(1 \mathrm{H}, \mathrm{d}$, $\left.J=8.4 \mathrm{~Hz}, \mathrm{H}-5^{\prime}\right), 6.35$ (1H, s, H-8), 6.15 (1H, s, H-6), $5.29(1 \mathrm{H}$, d, $J=4.2 \mathrm{~Hz}$, Glc H-1), 4.37 (1H, s, Rham H-1), 0.96 (3H, d, $J$ $=6.5 \mathrm{~Hz}$, Rham H-6); ${ }^{13} \mathrm{C}-\mathrm{NMR}\left(150 \mathrm{MHz}, \mathrm{DMSO}-d_{6}\right): 177.1$ (C-4), 164.9 (C-7), 160.8 (C-5), 156.5 (C-2, 9), 148.2 (C-4'), $144.5\left(\mathrm{C}-3^{\prime}\right), 133.2(\mathrm{C}-3), 121.6\left(\mathrm{C}-6^{\prime}\right), 121.1\left(\mathrm{C}-1^{\prime}\right), 116.0(\mathrm{C}-$ $5^{\prime}$ ), $115.1\left(\mathrm{C}-2^{\prime}\right), 103.5$ (C-10), 101.2 (Glc C-1), 100.6 (Rham C1), 98.9 (C-6), 93.8 (C-8), 76.2 (Glc C-3), 75.7 (Glc C-5), 73.9 (Glc C-2), 71.7 (Rham C-4), 70.4 (Rham C-2), 70.2 (Rham C-3), 69.8 (Glc C-4), 68.2 (Rham C-5), 67.0 (Glc C-6), 17.6 (Rham C-6).

Compound 12: $\mathrm{C}_{16} \mathrm{H}_{18} \mathrm{O}_{9}$; ESI/LTQ-Orbitrap-MS $\mathrm{m} / z$ : $376.26[\mathrm{M}+\mathrm{Na}]^{+} ;{ }^{1} \mathrm{H}-\mathrm{NMR}\left(600 \mathrm{MHz}, \mathrm{CD}_{3} \mathrm{OD}\right): 7.57(1 \mathrm{H}, \mathrm{d}$, $\left.J=15.6 \mathrm{~Hz}, \mathrm{H}-7^{\prime}\right), 6.97\left(1 \mathrm{H}, \mathrm{d}, J=1.8 \mathrm{~Hz}, \mathrm{H}-2^{\prime}\right), 6.86(1 \mathrm{H}$, $\left.\mathrm{dd}, J=1.8,7.8 \mathrm{~Hz}, \mathrm{H}-6^{\prime}\right), 6.71\left(1 \mathrm{H}, \mathrm{d}, J=7.8 \mathrm{~Hz}, \mathrm{H}-5^{\prime}\right), 6.25$ $\left(1 \mathrm{H}, \mathrm{d}, J=15.6 \mathrm{~Hz}, \mathrm{H}-8^{\prime}\right), 5.06(1 \mathrm{H}, \mathrm{m}, \mathrm{H}-3), 4.16(1 \mathrm{H}, \mathrm{m}, \mathrm{H}-$ 5), 3.82 (1H, m, H-4), 2.03-1.79 (4H, m, H-2, 6); ${ }^{13} \mathrm{C}-\mathrm{NMR}$ (150 MHz, CD 3 OD): 178.2 (1-COOH), $170.5\left({\mathrm{C}-9^{\prime}}^{\prime}\right), 151.4(\mathrm{C}-$ $\left.4^{\prime}\right), 149.3\left(\mathrm{C}-7^{\prime}\right), 148.9\left(\mathrm{C}-3^{\prime}\right), 129.6\left(\mathrm{C}-1^{\prime}\right), 125.0\left(\mathrm{C}-6^{\prime}\right), 118.4$ $\left(\mathrm{C}-5^{\prime}\right), 117.1\left(\mathrm{C}-2^{\prime}\right), 116.8\left(\mathrm{C}-8^{\prime}\right), 76.5(\mathrm{C}-1), 74.0$ (C-3), 72.6 (C-4), 71.2 (C-5), 39.6 (C-2), 39.2 (C-6).

Compound 13: $\mathrm{C}_{25} \mathrm{H}_{24} \mathrm{O}_{12}$; FAB-MS m/z: $517[\mathrm{M}+\mathrm{H}]^{+}$; ${ }^{1} \mathrm{H}-\mathrm{NMR}\left(600 \mathrm{MHz}, \mathrm{CD}_{3} \mathrm{OD}\right): 7.63\left(1 \mathrm{H}, \mathrm{d}, J=16.2 \mathrm{~Hz}, \mathrm{H}-7^{\prime \prime}\right)$, $7.56\left(1 \mathrm{H}, \mathrm{d}, J=16.2 \mathrm{~Hz}, \mathrm{H}-7^{\prime}\right), 7.08\left(1 \mathrm{H}, \mathrm{d}, J=4.2 \mathrm{~Hz}, \mathrm{H}-1^{\prime \prime}\right)$, $7.06\left(1 \mathrm{H}, \mathrm{d}, J=4.2 \mathrm{~Hz}, \mathrm{H}-1^{\prime}\right), 7.03\left(1 \mathrm{H}, \mathrm{d}, J=1.8 \mathrm{~Hz}, \mathrm{H}-2^{\prime}\right), 7.00$ $\left(1 \mathrm{H}, \mathrm{d}, J=1.8 \mathrm{~Hz}, \mathrm{H}-2^{\prime \prime}\right), 6.97\left(1 \mathrm{H}, \mathrm{dd}, J=1.8,6.6 \mathrm{~Hz}, \mathrm{H}-6^{\prime \prime}\right)$, $6.96\left(1 \mathrm{H}, \mathrm{d}, J=6.6 \mathrm{~Hz}, \mathrm{H}-5^{\prime \prime}\right), 6.80(1 \mathrm{H}, \mathrm{dd}, J=1.8,6.6 \mathrm{~Hz}$, H- $\left.6^{\prime}\right), 6.78\left(1 \mathrm{H}, \mathrm{d}, J=6.6 \mathrm{~Hz}, \mathrm{H}-5^{\prime}\right), 6.34(1 \mathrm{H}, \mathrm{d}, 2=16.2 \mathrm{~Hz}$, $\left.\mathrm{H}-8^{\prime \prime}\right), 6.26\left(1 \mathrm{H}, \mathrm{d}, J=16.2 \mathrm{~Hz}, \mathrm{H}-8^{\prime}\right), 5.44(1 \mathrm{H}, \mathrm{m}, \mathrm{H}-3), 4.31$ $(1 \mathrm{H}, \mathrm{d}, J=4.2 \mathrm{~Hz}, \mathrm{H}-5), 3.99(1 \mathrm{H}, \mathrm{dd}, J=3.7,7.2 \mathrm{~Hz}, \mathrm{H}-4)$, 2.33 (2H, m, H-6), 2.15 (2H, m, H-2); ${ }^{13} \mathrm{C}-\mathrm{NMR}(150 \mathrm{MHz}$, $\left.\mathrm{CD}_{3} \mathrm{OD}\right): 177.5$ (1-COOH), $169.0\left(\mathrm{C}-9^{\prime \prime}\right), 168.9\left(\mathrm{C}-9^{\prime}\right), 149.6$ $\left(\mathrm{C}-4^{\prime \prime}\right), 149.5\left(\mathrm{C}-4^{\prime}\right), 147.8\left(\mathrm{C}-3^{\prime \prime}\right), 147.4\left(\mathrm{C}-7^{\prime}, 7^{\prime \prime}\right), 146.2(\mathrm{C}-$ $\left.3^{\prime}\right), 128.0\left(\mathrm{C}-1^{\prime \prime}\right), 127.9\left(\mathrm{C}-1^{\prime}\right), 123.2\left(\mathrm{C}-6^{\prime \prime}\right), 123.1\left(\mathrm{C}-6^{\prime}\right), 116.6$ $\left(\mathrm{C}-5^{\prime}, 5^{\prime \prime}\right), 115.7\left(\mathrm{C}-8^{\prime \prime}\right), 115.4\left(\mathrm{C}-8^{\prime}\right), 115.3\left(\mathrm{C}-2^{\prime \prime}\right), 115.2\left(\mathrm{C}-2^{\prime}\right)$, 81.0 (C-1), 74.8 (C-4), 72.7 (C-3), 72.2 (C-5), 37.7 (C-6), 36.4 (C-2).

Compound 14: $\mathrm{C}_{25} \mathrm{H}_{24} \mathrm{O}_{12}$; Q-TOF-MS $m / z$ : 515.1185 $[\mathrm{M}-\mathrm{H}]^{-} ;{ }^{1} \mathrm{H}-\mathrm{NMR}\left(600 \mathrm{MHz}, \mathrm{CD}_{3} \mathrm{OD}\right): 7.60(1 \mathrm{H}, \mathrm{d}, J=$ $\left.15.6 \mathrm{~Hz}, \mathrm{H}-7^{\prime}\right), 7.52\left(1 \mathrm{H}, \mathrm{d}, J=15.6 \mathrm{~Hz}, \mathrm{H}-7^{\prime \prime}\right), 7.03(1 \mathrm{H}, \mathrm{d}, J$ $\left.=1.8 \mathrm{~Hz}, \mathrm{H}-2^{\prime}\right), 7.00\left(1 \mathrm{H}, \mathrm{d}, J=1.8 \mathrm{~Hz}, \mathrm{H}-2^{\prime \prime}\right), 6.92(2 \mathrm{H}, \mathrm{m}, \mathrm{H}-$ $\left.6^{\prime}, 6^{\prime \prime}\right), 6.76\left(1 \mathrm{H}, \mathrm{d}, J=7.8 \mathrm{~Hz}, \mathrm{H}-5^{\prime}\right), 6.74(1 \mathrm{H}, \mathrm{d}, J=7.8 \mathrm{~Hz}$, $\left.\mathrm{H}-5^{\prime \prime}\right), 6.29\left(1 \mathrm{H}, \mathrm{d}, J=15.6 \mathrm{~Hz}, \mathrm{H}-8^{\prime}\right), 6.20(1 \mathrm{H}, \mathrm{d}, J=15.6 \mathrm{~Hz}$, $\left.\mathrm{H}-8^{\prime \prime}\right), 5.65(1 \mathrm{H}, \mathrm{m}, \mathrm{H}-3), 5.12(1 \mathrm{H}, \mathrm{dd}, J=3.0,9.0 \mathrm{~Hz}, \mathrm{H}-4)$, $4.36(1 \mathrm{H}, \mathrm{d}, J=3.0 \mathrm{~Hz}, \mathrm{H}-5), 2.35(2 \mathrm{H}, \mathrm{m}, \mathrm{H}-6), 2.13(2 \mathrm{H}, \mathrm{m}$, $\mathrm{H}-2) ;{ }^{13} \mathrm{C}-\mathrm{NMR}$ (150 MHz, CD $\mathrm{CD}_{3}$ ): 177.2 (1-COOH), 170.6
$\left(\mathrm{C}-9^{\prime}\right), 170.3\left(\mathrm{C}-9^{\prime \prime}\right), 151.5\left(\mathrm{C}-4^{\prime}, 4^{\prime \prime}\right), 149.7\left(\mathrm{C}-7^{\prime}\right), 149.5(\mathrm{C}-$ $\left.7^{\prime \prime}\right), 148.6\left(\mathrm{C}-3^{\prime}, 3^{\prime \prime}\right), 129.6\left(\mathrm{C}-1^{\prime}\right), 129.5\left(\mathrm{C}-1^{\prime \prime}\right), 125.1\left(\mathrm{C}-6^{\prime}\right.$, $\left.6^{\prime \prime}\right), 118.4\left(\mathrm{C}-5^{\prime}, 5^{\prime \prime}\right), 117.1\left(\mathrm{C}-2^{\prime}, 2^{\prime \prime}\right), 116.6\left(\mathrm{C}-8^{\prime}, 8^{\prime \prime}\right), 78.0(\mathrm{C}-$ 1), 77.9 (C-4), 71.5 (C-5), 71.0 (C-3), 41.4 (C-2), 40.3 (C-6).

Compound 15: $\mathrm{C}_{25} \mathrm{H}_{24} \mathrm{O}_{12}$; Q-TOF-MS m/z: 515.1185 $[\mathrm{M}-\mathrm{H}]^{-} ;{ }^{1} \mathrm{H}-\mathrm{NMR}\left(600 \mathrm{MHz}, \mathrm{CD}_{3} \mathrm{OD}\right): 7.62(1 \mathrm{H}, \mathrm{d}, J=$ $\left.16.2 \mathrm{~Hz}, \mathrm{H}-7^{\prime}\right), 7.58\left(1 \mathrm{H}, \mathrm{d}, J=16.2 \mathrm{~Hz}, \mathrm{H}-7^{\prime \prime}\right), 7.07(2 \mathrm{H}, \mathrm{t}, J$ $\left.=1.8,4.2 \mathrm{~Hz}, \mathrm{H}-2^{\prime}, 2^{\prime \prime}\right), 6.96\left(2 \mathrm{H}, \mathrm{m}, \mathrm{H}-6^{\prime}, 6^{\prime \prime}\right), 6.75(1 \mathrm{H}, \mathrm{d}$, $\left.J=7.8 \mathrm{~Hz}, \mathrm{H}-5^{\prime}\right), 6.72\left(1 \mathrm{H}, \mathrm{d}, J=7.8 \mathrm{~Hz}, \mathrm{H}-5^{\prime \prime}\right), 6.36(1 \mathrm{H}, \mathrm{d}$, $\left.J=16.2 \mathrm{~Hz}, \mathrm{H}-8^{\prime}\right), 6.28\left(1 \mathrm{H}, \mathrm{d}, J=16.2 \mathrm{~Hz}, \mathrm{H}-8^{\prime \prime}\right), 5.41(1 \mathrm{H}$, m, H-3), 5.39 (1H, m, H-5), $3.98(1 \mathrm{H}, \mathrm{dd}, J=3.6,7.8 \mathrm{~Hz}, \mathrm{H}-4)$, 2.35 (2H, m, H-6), 2.20 (2H, m, H-2); ${ }^{13} \mathrm{C}-\mathrm{NMR}(150 \mathrm{MHz}$, $\left.\mathrm{CD}_{3} \mathrm{OD}\right): 179.4$ (1-COOH), $170.9\left(\mathrm{C}-9^{\prime}\right), 170.4\left(\mathrm{C}-9^{\prime \prime}\right), 151.5$ $\left(\mathrm{C}-4^{\prime}\right), 151.4\left(\mathrm{C}-4^{\prime \prime}\right), 149.2\left(\mathrm{C}-7^{\prime}, 7^{\prime \prime}\right), 149.0\left(\mathrm{C}-3^{\prime}\right), 148.6(\mathrm{C}-$ $\left.3^{\prime \prime}\right), 129.8\left(\mathrm{C}-1^{\prime}\right), 129.7\left(\mathrm{C}-1^{\prime \prime}\right), 125.0\left(\mathrm{C}-6^{\prime}\right), 124.9\left(\mathrm{C}-6^{\prime \prime}\right), 118.4$ $\left(\mathrm{C}-5^{\prime}, 5^{\prime \prime}\right), 117.5\left(\mathrm{C}-8^{\prime}\right), 117.2\left(\mathrm{C}-8^{\prime \prime}\right), 117.1\left(\mathrm{C}-2^{\prime}\right), 117.0\left(\mathrm{C}-2^{\prime \prime}\right)$, 76.7 (C-1), 74.5 (C-5), 74.0 (C-3), 72.6 (C-4), 39.7 (C-2), 38.0 (C-6).

Compound 16: $\mathrm{C}_{26} \mathrm{H}_{26} \mathrm{O}_{12}$; Q-TOF-MS $m / z$ : 529.1349 $[\mathrm{M}-\mathrm{H}]^{-} ;{ }^{1} \mathrm{H}-\mathrm{NMR}\left(600 \mathrm{MHz}, \mathrm{CD}_{3} \mathrm{OD}\right): 7.63(1 \mathrm{H}, \mathrm{d}, J=$ $\left.16.2 \mathrm{~Hz}, \mathrm{H}-7^{\prime}\right), 7.56\left(1 \mathrm{H}, \mathrm{d}, J=16.2 \mathrm{~Hz}, \mathrm{H}-7^{\prime \prime}\right), 7.07(1 \mathrm{H}, \mathrm{d}$, $\left.J=2.4 \mathrm{~Hz}, \mathrm{H}-2^{\prime}\right), 7.06\left(1 \mathrm{H}, \mathrm{d}, J=2.4 \mathrm{~Hz}, \mathrm{H}-2^{\prime \prime}\right), 6.97(2 \mathrm{H}$, m, H-6', $\left.6^{\prime \prime}\right), 6.80\left(1 \mathrm{H}, \mathrm{d}, J=8.4 \mathrm{~Hz}, \mathrm{H}-5^{\prime}\right), 6.78(1 \mathrm{H}, \mathrm{d}, J$ $\left.=8.4 \mathrm{~Hz}, \mathrm{H}-5^{\prime \prime}\right), 6.35\left(1 \mathrm{H}, \mathrm{d}, J=16.2 \mathrm{~Hz}, \mathrm{H}-8^{\prime}\right), 6.23(1 \mathrm{H}$, d, $\left.J=16.2 \mathrm{~Hz}, \mathrm{H}-8^{\prime \prime}\right), 5.41(1 \mathrm{H}, \mathrm{m}, \mathrm{H}-3), 5.32(1 \mathrm{H}, \mathrm{m}, \mathrm{H}-5)$, $3.98(1 \mathrm{H}, \mathrm{dd}, J=3.0,6.6 \mathrm{~Hz}, \mathrm{H}-4), 3.69$ (3H, s, 1-COOMe), 2.35 (2H, m, H-6), 2.20 (2H, m, H-2); ${ }^{13} \mathrm{C}-\mathrm{NMR}(150 \mathrm{MHz}$,

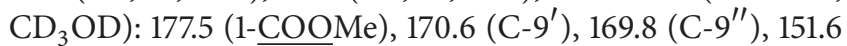
$\left(\mathrm{C}-4^{\prime}\right), 151.4\left(\mathrm{C}-4^{\prime \prime}\right), 149.3\left(\mathrm{C}-7^{\prime}\right), 149.0\left(\mathrm{C}-7^{\prime \prime}\right), 148.7\left(\mathrm{C}-3^{\prime}\right)$, $148.6\left(\mathrm{C}-3^{\prime \prime}\right), 129.7\left(\mathrm{C}-1^{\prime}\right), 129.4\left(\mathrm{C}-1^{\prime \prime}\right), 124.9\left(\mathrm{C}-6^{\prime}\right), 124.8(\mathrm{C}-$ $\left.6^{\prime \prime}\right), 118.4\left(\mathrm{C}-5^{\prime}\right), 118.3\left(\mathrm{C}-5^{\prime \prime}\right), 117.2\left(\mathrm{C}-8^{\prime}, 8^{\prime}\right), 117.0\left(\mathrm{C}-2^{\prime}, 2^{\prime}\right)$ 76.5 (C-1), 74.0 (C-5), 73.8 (C-3), 72.6 (C-4), 54.9 (1-COOMe), 38.5 (C-2), 37.5 (C-6).

Compound 17: $\mathrm{C}_{26} \mathrm{H}_{44} \mathrm{O}_{8}$; ESI/LTQ-Orbitrap-MS $\mathrm{m} / z$ : $507.29[\mathrm{M}+\mathrm{Na}]^{+} ;{ }^{1} \mathrm{H}-\mathrm{NMR}\left(600 \mathrm{MHz}\right.$, Pyridine- $\left.d_{6}\right): 5.06$ $(1 \mathrm{H}, \mathrm{d}, J=8.4 \mathrm{~Hz}$, Glc H-1), $4.52(1 \mathrm{H}, \mathrm{d}, J=10.6 \mathrm{~Hz}, \mathrm{H}-17 \mathrm{a})$, $4.12(1 \mathrm{H}, \mathrm{t}, \mathrm{H}-3), 3.95(1 \mathrm{H}, \mathrm{d}, J=10.6 \mathrm{~Hz}, \mathrm{H}-17 \mathrm{~b}), 2.43(1 \mathrm{H}, \mathrm{s}$, H-14a), 2.02 (1H, m, H-13), 1.94 (1H, m, H-2a), 1.88 (1H, m, H-1a), 1.82 (1H, m, H-15a), 1.78 (1H, m, H-14b), 1.66 (1H, m, H-7a), 1.65 (2H, m, H-11), 1.64 (1H, m, H-6a), 1.57 (1H, m, H5), 1.47 (1H, m, H-7b), 1.42 (1H, m, H-6b), 1.35 (1H, m, 15b), $1.34(1 \mathrm{H}, \mathrm{m}, \mathrm{H}-2 \mathrm{~b}), 1.21(3 \mathrm{H}, \mathrm{s}, 20), 1.02(3 \mathrm{H}, \mathrm{s}, 19), 0.99(3 \mathrm{H}, \mathrm{s}$, 18), 0.98 (1H, m, H-9), 0.89 (1H, m, 1b); ${ }^{13} \mathrm{C}-\mathrm{NMR}(150 \mathrm{MHz}$, pyridine- $d_{6}$ ): 105.7 (Glc C-1), 82.5 (C-16), 80.1 (C-3), 78.4 (Glc C-5), 78.3 (Glc C-3), 75.7 (Glc C-2), 75.5 (C-17), 72.1 (Glc C4), 63.2 (Glc C-6), 58.6 (C-9), 56.9 (C-5), 54.0 (C-15), 47.2 (C13), 46.0 (C-8), 43.6 (C-7), 40.6 (C-10), 40.4 (C-4), 40.3 (C-1), 38.5 (C-14), 29.3 (C-19), 28.4 (C-2), 27.7 (C-12), 21.7 (C-6), 19.9 (C-11), 18.8 (C-20), 16.6 (C-18).

Compound 18: $\mathrm{C}_{26} \mathrm{H}_{44} \mathrm{O}_{8}$; ESI/LTQ-Orbitrap-MS $\mathrm{m} / z$ : $507.29[\mathrm{M}+\mathrm{Na}]^{+} ;{ }^{1} \mathrm{H}-\mathrm{NMR}\left(600 \mathrm{MHz}\right.$, Pyridine- $\left.d_{6}\right): 5.05(1 \mathrm{H}$, d, $J=7.8 \mathrm{~Hz}$, Glc H-1), $4.49(1 \mathrm{H}, \mathrm{d}, J=10.6 \mathrm{~Hz}, \mathrm{H}-17 \mathrm{a}), 4.12$ (1H, t, H-3), 3.92 (1H, d, J = 10.6 Hz, H-17b), 2.45 (1H, s, H14a), 2.02 (1H, m, H-13), 1.94 (1H, m, H-2a), 1.88 (1H, m, H1a), 1.82 (1H, m, H-15a), 1.78 (1H, m, H-14b), 1.66 (1H, m, H7a), 1.65 (2H, m, H-11), 1.64 (1H, m, H-6a), 1.57 (1H, m, H-5), 1.47 (1H, m, H-7b), 1.42 (1H, m, H-6b), 1.35 (1H, m, 15b), 1.34 (1H, m, H-2b), 1.18 (3H, s, 20), 1.02 (3H, s, 19), 0.98 (1H, m, $\mathrm{H}-9), 0.89$ (1H, m, 1b), 0.88 (3H, s, 18); ${ }^{13} \mathrm{C}-\mathrm{NMR}(150 \mathrm{MHz}$, 
TABLE 1: $\mathrm{IC}_{50}$ of the extraction and fractions from A. iwayomogi on aldose reductase (AR) and advanced glycation endproducts (AGEs) formation.

\begin{tabular}{|c|c|c|}
\hline \multirow{2}{*}{ Sample } & \multicolumn{2}{|c|}{$\mathrm{IC}_{50}{ }^{a}(\mu \mathrm{g} / \mathrm{ml})$} \\
\hline & $\mathrm{AR}$ & AGEs formation \\
\hline $50 \%$ EtOH ext. & $2.71 \pm 0.72^{* *}$ & $40.99 \pm 0.50^{*}$ \\
\hline $\mathrm{CHCl}_{3}$ fr. & $15.36 \pm 3.64^{* *}$ & $7.29 \pm 0.05^{*}$ \\
\hline $30 \% \mathrm{MeOH}$ fr. & $1.16 \pm 0.10^{*}$ & $40.81 \pm 0.05^{*}$ \\
\hline $60 \% \mathrm{MeOH}$ fr. & $1.42 \pm 0.17^{*}$ & $93.76 \pm 0.06^{*}$ \\
\hline $100 \% \mathrm{MeOH}$ fr. & $0.79 \pm 0.15^{*}$ & $38.40 \pm 0.51^{*}$ \\
\hline Water fr. & $19.67 \pm 0.81^{* *}$ & $402.45 \pm 4.28^{* *}$ \\
\hline $\mathrm{TMG}^{b}$ & $1.22 \pm 0.03^{*}$ & - \\
\hline $\mathrm{AG}^{c}$ & - & $45.85 \pm 7.17^{* *}$ \\
\hline
\end{tabular}

Pyridine- $d_{6}$ ): 107.4 (Glc C-1), 81.5 (C-16), 79.4 (Glc C-5), 79.3 (Glc C-3), 76.4 (Glc C-2), 76.2 (C-17), 75.8 (C-3), 72.3 (Glc C-4), 63.5 (Glc C-6), 57.5 (C-9), 54.0 (C-15), 49.6 (C-5), 47.2 (C-13), 45.5 (C-8), 43.1 (C-7), 40.2 (C-10), 38.7 (C-4), 38.2 (C14), 34.5 (C-1), 30.1 (C-18), 27.6 (C-2), 27.1 (C-12), 23.1 (C-19), 21.1 (C-6), 19.2 (C-20), 18.6 (C-11).

Compound 19: $\mathrm{C}_{26} \mathrm{H}_{42} \mathrm{O}_{8}$; FAB-MS m/z: $481[\mathrm{M}-\mathrm{H}]^{-}$; ${ }^{1} \mathrm{H}-\mathrm{NMR}\left(600 \mathrm{MHz}, \mathrm{DMSO}-d_{6}\right): 4.16(1 \mathrm{H}, \mathrm{d}, J=7.8 \mathrm{~Hz}$, Glc $\mathrm{H}-1), 4.03(1 \mathrm{H}, \mathrm{d}, J=10.8 \mathrm{~Hz}, \mathrm{H}-17 \mathrm{a}), 3.38(1 \mathrm{H}, \mathrm{d}, J=10.8 \mathrm{~Hz}$, $\mathrm{H}-17 \mathrm{a}), 2.43$ (1H, m, H-2a), 1.99 (1H, m, H-13), 1.92 (1H, m, $\mathrm{H}-1), 1.82$ (1H, m, H-2b), 1.77 (2H, m, H-14), 1.58 (1H, m, H5), 1.57 (1H, m, H-15a), 1.56 (2H, m, H-11), 1.51 (2H, m, H-12), 1.45 (2H, m, H-7), 1.40 (2H, m, H-6), 1.35 (1H, m, H-15b), 1.07 (1H, m, H-9), 0.99 (6H, s, H-18, 20), $0.93(3 \mathrm{H}, \mathrm{s}, \mathrm{H}-19) ;{ }^{13} \mathrm{C}-$ NMR (150 MHz, DMSO- $d_{6}$ ): 218.7 (C-3), 105.8 (Glc C-1), 81.2 (C-16), 81.0 (Glc C-5), 77.6 (Glc C-3), 75.3 (Glc C-2), 75.1 (C17), 71.5 (Glc C-4), 62.5 (Glc C-6), 56.3 (C-9), 54.8 (C-5), 53.2 (C-15), 48.0 (C-4), 46.3 (C-13), 45.3 (C-8), 42.1 (C-7), 40.1 (C1), 39.5 (C-10), 37.8 (C-14), 35.1 (C-2), 28.4 (C-18), 27.3 (C-12), 22.8 (C-6), 22.2 (C-19), 19.9 (C-11), 19.0 (C-20).

Compound 20: $\mathrm{C}_{15} \mathrm{H}_{20} \mathrm{O}_{9}$; FAB-MS $m / z$ : $335[\mathrm{M}+\mathrm{H}]^{+}$; ${ }^{1} \mathrm{H}-\mathrm{NMR}\left(600 \mathrm{MHz}, \mathrm{CD}_{3} \mathrm{OD}\right): 6.27(1 \mathrm{H}, \mathrm{d}, J=2.4 \mathrm{~Hz}, \mathrm{H}-$ 5), $6.19(1 \mathrm{H}, \mathrm{d}, J=2.4 \mathrm{~Hz}, \mathrm{H}-3), 5.04(1 \mathrm{H}, \mathrm{d}, J=7.8 \mathrm{~Hz}$, Glc $\mathrm{H}-1), 3.89$ (3H, s, 6-OMe), $2.59\left(3 \mathrm{H}, \mathrm{s}, \mathrm{COCH}_{3}\right) ;{ }^{13} \mathrm{C}-\mathrm{NMR}$ $\left(150 \mathrm{MHz}, \mathrm{CD}_{3} \mathrm{OD}\right): 206.2\left(\mathrm{COCH}_{3}\right), 166.7(\mathrm{C}-2), 165.2(\mathrm{C}-$ 4), 164.8 (C-6), 107.9 (C-1), 100.8 (Glc C-1), 97.4 (C-3), 93.2 (C-5), 77.9 (Glc C-3), 77.2 (Glc C-5), 74.3 (Glc C-2), 70.9 (Glc C-4), 62.0 (Glc C-6), 56.7 (6-OMe), $33.3\left(\mathrm{COCH}_{3}\right)$.

3.2. AR Inhibitory Activities of the Extracts, Fractions, and Compounds 1-20 from A. iwayomogi. Our study investigated the inhibitory effects of $A$. iwayomogi on AR and AGEs formation, which demonstrates the potential of these compounds to prevent diabetes complications. The extracts and fractions of the aerial parts of the A. iwayomogi were tested for AR inhibition. The results are summarized in Table 1 . The extracts, $30 \%, 60 \%$, and $100 \% \mathrm{MeOH}$ fractions from A. iwayomogi showed significant inhibition of AR
TABLE 2: $\mathrm{IC}_{50}$ of compounds $\mathbf{1 - 2 0}$ isolated from A. iwayomogi on aldose reductase (AR) and advanced glycation endproducts (AGEs) formation.

\begin{tabular}{lcc}
\hline Compound & \multicolumn{2}{c}{$\mathrm{IC}_{50}{ }^{a}(\mu \mathrm{M})$} \\
\hline 1 & $90.39 \pm 1.91^{* *}$ & AGEs formation \\
$\mathbf{2}$ & $131.37 \pm 0.12^{*}$ & $20.71 \pm 1.38^{* *}$ \\
$\mathbf{3}$ & $>200$ & $82.43 \pm 1.52^{* *}$ \\
$\mathbf{4}$ & $2.93 \pm 0.11^{* *}$ & $311.89 \pm 11.26^{* *}$ \\
$\mathbf{5}$ & $110.65 \pm 9.89^{* *}$ & $185.56 \pm 0.20^{* *}$ \\
$\mathbf{6}$ & $30.29 \pm 3.95^{*}$ & $169.61 \pm 16.41^{* *}$ \\
$\mathbf{7}$ & $102.53 \pm 7.81^{* *}$ & $134.88 \pm 0.84^{* *}$ \\
$\mathbf{8}$ & $3.19 \pm 0.10^{* *}$ & $207.70 \pm 13.32^{* *}$ \\
$\mathbf{9}$ & $3.32 \pm 0.21^{* *}$ & $197.38 \pm 11.03^{* *}$ \\
$\mathbf{1 0}$ & $10.60 \pm 0.38^{* *}$ & $67.42 \pm 9.95^{* *}$ \\
$\mathbf{1 1}$ & $12.53 \pm 0.20^{* *}$ & $109.46 \pm 1.41^{* *}$ \\
$\mathbf{1 2}$ & $4.54 \pm 0.16^{* *}$ & $129.15 \pm 4.01^{* *}$ \\
$\mathbf{1 3}$ & $0.22 \pm 0.01^{* *}$ & $176.16 \pm 5.77^{* *}$ \\
$\mathbf{1 4}$ & $1.90 \pm 0.01^{* *}$ & $24.85 \pm 1.86^{* *}$ \\
$\mathbf{1 5}$ & $1.25 \pm 0.01^{* *}$ & $124.28 \pm 4.25^{* *}$ \\
$\mathbf{1 6}$ & $1.48 \pm 0.04^{* *}$ & $31.78 \pm 0.56^{* *}$ \\
$\mathbf{1 7}$ & $>200$ & $44.45 \pm 4.86^{* *}$ \\
$\mathbf{1 8}$ & $>200$ & $\mathrm{ND}^{d}$ \\
$\mathbf{1 9}$ & $>200$ & $\mathrm{ND}^{d}$ \\
$\mathbf{2 0}$ & $>200$ & $\mathrm{ND}^{d}$ \\
$\mathrm{TMG}^{b}$ & $-5.5 \pm 0.19^{*}$ & - \\
$\mathrm{AG}^{c}$ & - & $414.77 \pm 5.96^{*}$ \\
\hline & & $048.99 \pm 0.11^{*}$ \\
\hline
\end{tabular}

${ }^{a} \mathrm{IC}_{50}$ calculated from the least-squares regression line of the logarithmic concentrations plotted against the residual activity.

${ }^{b}$ TMG was used as a positive control.

${ }^{c}$ AG was used as a positive control.

${ }^{d}$ ND was not detected.

${ }^{*}$ Significant difference from control; ${ }^{*} p<0.05,{ }^{* *} p<0.01$.

$\left(\mathrm{IC}_{50}\right.$ value with $2.71 \pm 0.72,1.16 \pm 0.10,1.42 \pm 0.17$, and $0.79 \pm 0.15 \mu \mathrm{g} / \mathrm{mL}$, resp.). In addition, compounds 1-20 were tested for their ability to inhibit AR. As can be seen in Table 2, genkwanin (3), the three diterpene glycosides (17-19), and 2,4-dihydroxy 6-methoxyacetophenone 4-O- $\beta$ D-glucopyranoside (20) demonstrated no AR inhibition; all had $\mathrm{IC}_{50}$ values $>200 \mu \mathrm{M}$. On the other hand, hispidulin (4), patuletin-3-O-glucoside (8), isoquercetin (9), and five caffeoylquinic acids $(12,13,14,15$, and 16) were stronger inhibitors than TMG $\left(\mathrm{IC}_{50}\right.$ value with $\left.6.58 \pm 0.19 \mu \mathrm{M}\right)$, which was used as the positive control, as a compound known to inhibit AR. The $\mathrm{IC}_{50}$ values of compounds 4, 8, 9, 12, 13, 14, 15, and 16 were $2.93 \pm 0.11,3.19 \pm 0.10,3.32 \pm 0.21,4.54 \pm 0.16,0.22$ $\pm 0.01,1.90 \pm 0.01,1.25 \pm 0.01$, and $1.48 \pm 0.04 \mu \mathrm{M}$, respectively.

3.3. Inhibitory Activities of the Extracts, Fractions, and Compounds 1-20 from A. iwayomogi on AGEs Formation. The extracts and fractions from $A$. iwayomogi were investigated for inhibition of AGEs formation. The results are shown in Table 1. The extracts, $\mathrm{CHCl}_{3}, 30 \% \mathrm{MeOH}$, and $100 \% \mathrm{MeOH}$ fractions all showed greater inhibition than $\mathrm{AG}\left(\mathrm{IC}_{50}\right.$ value with $45.85 \pm 7.17 \mu \mathrm{g} / \mathrm{mL}$ ), the positive control. The $\mathrm{IC}_{50}$ values 
TABLE 3: Contents of compounds $\mathbf{1}, \mathbf{2}, \mathbf{8}, \mathbf{1 0}, \mathbf{1 1}, \mathbf{1 2}, \mathbf{1 4}$, and 15 in A. iwayomogi by region in Korea.

\begin{tabular}{|c|c|c|c|c|c|c|c|c|}
\hline \multirow{2}{*}{ Sample } & \multicolumn{8}{|c|}{ Contents (mg/g) } \\
\hline & 1 & 2 & 8 & 10 & 11 & 12 & 14 & 15 \\
\hline Seoul & $1.38 \pm 0.01$ & $1.16 \pm 0.02$ & $.80 \pm 0.03$ & $25 \pm 0.20$ & $0.69 \pm 0.42$ & $16.79 \pm 0.19$ & $7.43 \pm 0.18$ & $55.86 \pm 0.79$ \\
\hline Gyeo & $8 \pm 0.01$ & $19 \pm 0.01$ & $4 \pm 0.03$ & $23 \pm 0.07$ & $.23 \pm 0.01$ & $4.18 \pm 0.05$ & $3.40 \pm 0.01$ & $30.83 \pm 0.06$ \\
\hline Jechon, Chungcheo & $0.57 \pm 0.10$ & $0.43 \pm 0.01$ & $1.25 \pm 0.06$ & $0.22 \pm 0.02$ & $0.27 \pm 0.01$ & $5.62 \pm 0.05$ & $2.90 \pm 0.02$ & $32.44 \pm 0.28$ \\
\hline Imsil & $0.26 \pm 0.01$ & $0.30 \pm 0.01$ & $0.96 \pm 0.04$ & $0.14 \pm 0.02$ & $0.38 \pm 0.04$ & $2.56 \pm 0.22$ & $2.23 \pm 0.03$ & $17.82 \pm 0.07$ \\
\hline Jinan, Jeol & $0.96 \pm 0.04$ & $0.70 \pm 0.01$ & $1.85 \pm 0.01$ & $0.36 \pm 0.02$ & $0.25 \pm 0.04$ & $5.46 \pm 0.17$ & $4.11 \pm 0.03$ & $27.51 \pm 0.43$ \\
\hline Hwasun, Jeonllanam-do & $0.19 \pm 0.01$ & $0.62 \pm 0.01$ & $1.99 \pm 0.09$ & $0.26 \pm 0.03$ & $0.32 \pm 0.02$ & $1.66 \pm 0.14$ & $1.68 \pm 0.01$ & $14.33 \pm 0.64$ \\
\hline Gyeongju, Gyeongsang & $63 \pm 0.10$ & $0.75 \pm 0.03$ & $3.45 \pm 0.11$ & $0.65 \pm 0.06$ & $0.34 \pm 0.05$ & $15.28 \pm 0.15$ & $7.58 \pm 0.21$ & $55.49 \pm 0.17$ \\
\hline Uiseong, Gyeongsang & $0.50 \pm 0.01$ & $0.61 \pm 0.03$ & $1.27 \pm 0.02$ & $0.07 \pm 0.05$ & $0.43 \pm 0.26$ & $5.45 \pm 0.23$ & $1.63 \pm 0.01$ & $25.44 \pm 0.05$ \\
\hline Andong, Gyeongsangbuk-do & $0.94 \pm 0.01$ & $0.98 \pm 0.01$ & $2.34 \pm 0.02$ & $0.41 \pm 0.11$ & $0.43 \pm 0.01$ & $8.94 \pm 0.06$ & $4.49 \pm 0.03$ & $40.52 \pm 0.13$ \\
\hline Yeongcheon, Gyeongsangbuk-do & $0.29 \pm 0.01$ & $0.43 \pm 0.01$ & $1.21 \pm 0.03$ & $0.10 \pm 0.03$ & $0.51 \pm 0.02$ & $4.32 \pm 0.15$ & $1.94 \pm 0.01$ & $21.99 \pm 0.27$ \\
\hline Seongju, Gyeongsangbuk-do & $0.73 \pm 0.03$ & $0.29 \pm 0.01$ & $1.18 \pm 0.01$ & $0.20 \pm 0.02$ & $0.27 \pm 0.01$ & $3.07 \pm 0.09$ & $2.36 \pm 0.03$ & $21.92 \pm 0.07$ \\
\hline Gunwi, Gyeongsangbuk-do & $0.67 \pm 0.01$ & $0.75 \pm 0.01$ & $1.20 \pm 0.01$ & $0.20 \pm 0.03$ & $0.18 \pm 0.01$ & $2.79 \pm 0.07$ & $2.58 \pm 0.04$ & $20.46 \pm 0.01$ \\
\hline Sancheong, Gyeongsangnam-do & $1.05 \pm 0.01$ & $1.56 \pm 0.02$ & $3.25 \pm 0.02$ & $0.31 \pm 0.06$ & $0.39 \pm 0.04$ & $15.94 \pm 0.49$ & $3.46 \pm 0.08$ & $43.47 \pm 0.05$ \\
\hline Jeju-do & $0.12 \pm 0.03$ & $1.59 \pm 0.02$ & $4.03 \pm 0.10$ & $0.35 \pm 0.10$ & $1.11 \pm 0.07$ & $17.45 \pm 0.19$ & $5.34 \pm 0.03$ & $48.22 \pm 0.95$ \\
\hline Yangyang, Gangwon-do & $0.74 \pm 0.02$ & $1.67 \pm 0.02$ & $3.95 \pm 0.45$ & $0.59 \pm 0.12$ & $0.53 \pm 0.02$ & $33.61 \pm 0.48$ & $8.18 \pm 0.10$ & $68.87 \pm 0.19$ \\
\hline
\end{tabular}

Data are mean $\pm \mathrm{SD}(n=3) \mathrm{in} \mathrm{mg} / \mathrm{g}$ dried sample.

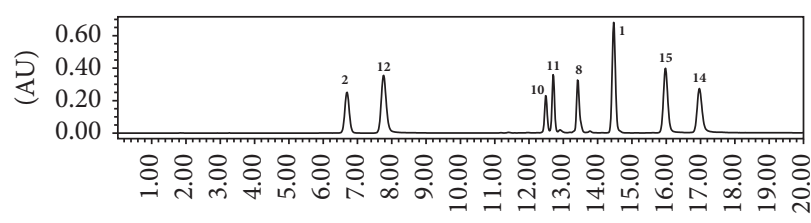

(Minutes)

(a)

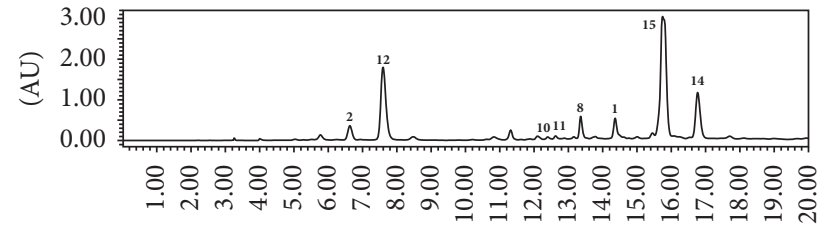

(Minutes)

(b)

Figure 2: Chromatograms of standards mixture (a) and A. iwayomogi ext. (b).

of extracts, $\mathrm{CHCl}_{3}, 30 \% \mathrm{MeOH}$, and $100 \% \mathrm{MeOH}$ fractions were $40.99 \pm 0.50,7.29 \pm 0.05,40.81 \pm 0.05$, and $38.40 \pm$ $0.51 \mu \mathrm{g} / \mathrm{mL}$, respectively. Compounds 1-20 isolated from $A$. iwayomogi were also tested for inhibition of AGEs formation, as reported in Table 2. Similar to AR inhibitory activities, diterpene glycosides (17-19) showed no inhibition of AGEs formation and compound $\mathbf{2 0}$ showed only slight inhibition, whereas the two coumarins (1 and 2), nine flavonoids (3-11), and five caffeoylquinic acids (12-16) were all more inhibitory than $\mathrm{AG}\left(\mathrm{IC}_{50}\right.$ value with $\left.414.77 \pm 5.96 \mu \mathrm{M}\right)$. Among them, compound 1, which exhibited minor inhibition on $A R$, exhibited the best inhibition of AGEs formation with an $\mathrm{IC}_{50}$ value of $20.71 \pm 1.38 \mu \mathrm{M}$.

3.4. Simultaneous Quantitative Analysis of Bioactive Compounds in A. iwayomogi to Select Suitable Region in Korea. Since A. iwayomogi is found mainly in Korea, we collected 15 samples of $A$. iwayomogi in different regions including Seoul, Gyeongii-do, Chungcheongbuk-do, Jeollabukdo, Jeollanam-do, Gyeongsangbuk-do, Gyeongsangnam-do, Gangwon-do, and Jeju-do. The following compounds were analyzed by HPLC-PAD from these fifteen specimens: scopoletin (1), scopolin (2), patulein-3-O-glucoside (8), quercetin-3-gentiobioside (10), rutin (11), 3-O-caffeoylquinic acid (12), 3,4-di-O-caffeoylquinic acid (14), and 3,5-di-Ocaffeoylquinic acid (15). They all showed significant inhibition of either AR or AGEs formation. Compounds 1, 2, 8, 10, $11,12,14$, and 15 were detected in $14.4,6.6,13.3,12.4,12.7$, 7.6, 16.9, and $15.9 \mathrm{~min}$, respectively (Figure 2). To optimize analytical methods, the standard calibration curve, limit of detection (LOD), and limit of quantification (LOQ) for compounds $1,2,8,10,11,12,14$, and 15 are measured. The standard calibration curves of compounds $1,2,8,10,11,12,14$, and 15 were detected at linear range $0.1-1000 \mu \mathrm{g} / \mathrm{mL}$. As a result, the correlation coefficient $\left(r^{2}\right)$ of all bioactive compounds is above 0.999 . The values of LOD of compounds $\mathbf{1 , 2}, \mathbf{8}, \mathbf{1 0}, \mathbf{1 1}$, 12,14 , and 15 are shown to be $2.80,0.50,5.93,5.08,4.99,3.82$, 1.81 , and 2.38 and the values of LOQ of bioactive compounds are shown to be $8.49,1.52,17.98,15.41,15.12,11.59,5.48$, and $7.21 \mu \mathrm{g} / \mathrm{mL}$, respectively. Using optimized analytical methods, the amounts of bioactive compounds from A. iwayomogi in the 15 samples were determined (Table 3 ). As a result, contents of compounds $\mathbf{1}$ and $\mathbf{8}$ were the highest in Seoul (1.38 \pm $0.01,4.80 \pm 0.03 \mathrm{mg} / \mathrm{g}$, resp.), compound 10 was the highest in Gyeongju, Gyeongsangbuk-do $(0.65 \pm 0.06 \mathrm{mg} / \mathrm{g})$, and compound 11 was highest in Jeju-do $(1.11 \pm 0.07 \mathrm{mg} / \mathrm{g})$. Furthermore, compounds 2, 12, 14, and 15 were the most abundant in Yangyang, Gangwon-do $(1.67 \pm 0.02,33.61 \pm 0.48$, 


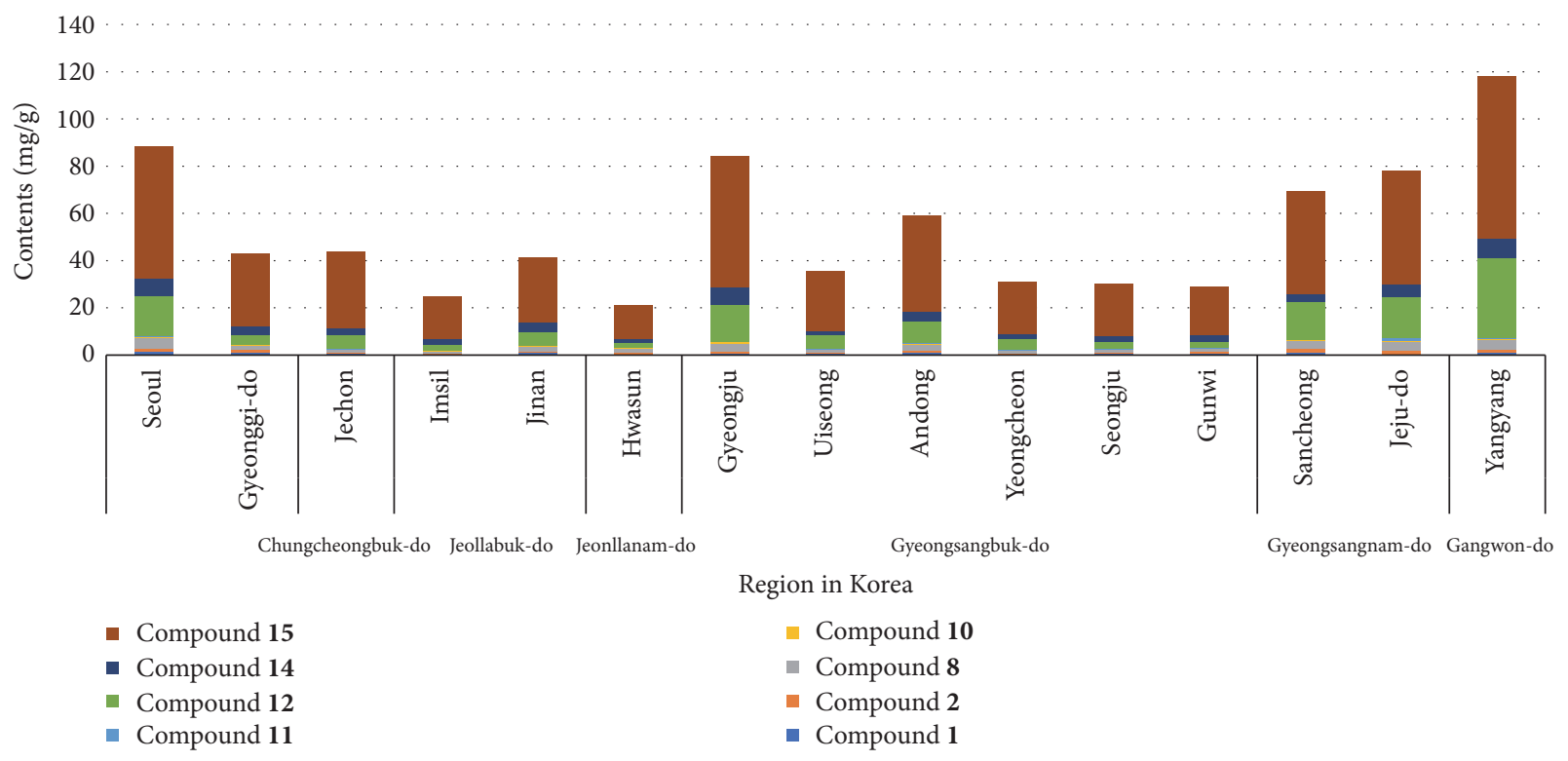

(a)

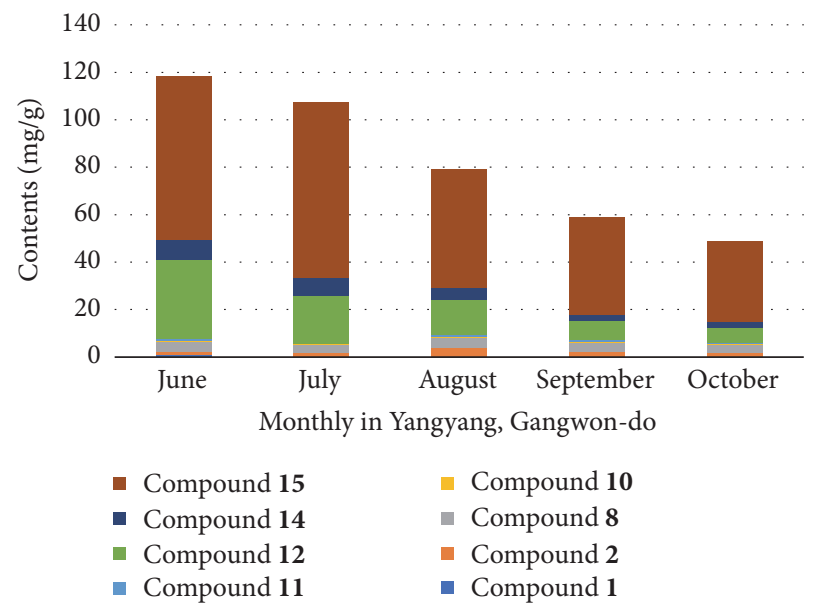

(b)

Figure 3: Total contents of compounds $1,2, \mathbf{8}, \mathbf{1 0}, \mathbf{1 1}, \mathbf{1 2}, \mathbf{1 4}$, and 15 in A. iwayomogi by region (a) and by month in Yangyang, Gangwon-do, Korea (b).

$8.18 \pm 0.10$, and $68.87 \pm 0.19 \mathrm{mg} / \mathrm{g}$, resp.). Compound 2 exhibited good inhibition activity of AGEs formation while compounds 12, 14, and 15 were significant inhibitors of both AR and AGEs formation. Overall, the specimen with the highest total contents of the most compounds was acquired in Yangyang, Gangwon-do (Figure 3). We therefore collected new specimens of A. iwayomogi in June, July, August, September, and October in Yangyang, Gangwon-do, and then analyzed the amounts of bioactive compounds in each specimen using HPLC (Table 4). Amounts of compounds $\mathbf{1}$, 10, 12, and 14 were taken in June $(0.74 \pm 0.02,0.59 \pm 0.12,33.61$ \pm 0.48 , and $8.18 \pm 0.10 \mathrm{mg} / \mathrm{g}$, resp.), that of compound 15 was taken in July $(73.99 \pm 0.35 \mathrm{mg} / \mathrm{g})$, those of compounds $\mathbf{2}$ and $\mathbf{8}$ were taken in August (3.67 \pm 0.02 and $4.16 \pm 0.04 \mathrm{mg} / \mathrm{g}$, resp.), and that of compound $\mathbf{1 1}$ was taken in September $(0.86 \pm$ $0.02 \mathrm{mg} / \mathrm{g}$ ).

\section{Discussion}

The worldwide prevalence of diabetes has become a massive health burden significantly decreasing quality of life and increasing morbidity, all at a huge economic cost. As diabetes is recognized as a serious disease, diabetic complications are also recognized as a serious disease. Accordingly, preventing treatment for diabetic complications is as important as diabetes and blocking the polyol pathway plays an important role preventing diabetic complications. Therefore, AR and AGEs formation inhibitors significantly affected diabetic complications. Artemisia iwayomogi, locally called haninjin or dowijigi, is a member of the Compositae family and a perennial herb mainly found in Korea. A. iwayomogi has long been used in Korea in foods such as tea, rice cake, and soup and for the treatment of various diseases. However, previous 
TABle 4: Contents of compounds $\mathbf{1}, \mathbf{2}, \mathbf{8}, \mathbf{1 0}, \mathbf{1 1}, \mathbf{1 2}, \mathbf{1 4}$, and 15 from A. iwayomogi in Yangyang, Gangwon-do by month.

\begin{tabular}{|c|c|c|c|c|c|c|c|c|}
\hline \multirow{2}{*}{ Sample } & \multicolumn{8}{|c|}{ Contents (mg/g) } \\
\hline & 1 & 2 & 8 & 10 & 11 & 12 & 14 & 15 \\
\hline June & $0.74 \pm 0.02$ & $1.67 \pm 0.02$ & $3.95 \pm 0.45$ & $0.59 \pm 0.12$ & $0.53 \pm 0.02$ & $33.61 \pm 0.48$ & $8.18 \pm 0.10$ & $68.87 \pm 0.19$ \\
\hline July & $0.08 \pm 0.01$ & $1.81 \pm 0.01$ & $3.19 \pm 0.08$ & $0.31 \pm 0.04$ & $0.47 \pm 0.01$ & $19.74 \pm 0.08$ & $7.73 \pm 0.02$ & $73.99 \pm 0.35$ \\
\hline August & $0.28 \pm 0.01$ & $3.67 \pm 0.02$ & $4.16 \pm 0.04$ & $0.30 \pm 0.04$ & $0.67 \pm 0.09$ & $15.15 \pm 0.01$ & $4.97 \pm 0.08$ & $49.99 \pm 0.99$ \\
\hline September & $0.20 \pm 0.01$ & $2.24 \pm 0.01$ & $3.71 \pm 0.01$ & $0.21 \pm 0.02$ & $0.86 \pm 0.02$ & $7.81 \pm 0.13$ & $2.88 \pm 0.01$ & $40.97 \pm 0.50$ \\
\hline October & $0.19 \pm 0.01$ & $1.58 \pm 0.01$ & $3.42 \pm 0.06$ & $0.30 \pm 0.02$ & $0.34 \pm 0.01$ & $6.63 \pm 3.07$ & $2.25 \pm 0.01$ & $34.03 \pm 0.09$ \\
\hline
\end{tabular}

Data are mean $\pm \mathrm{SD}(n=3)$ in $\mathrm{mg} / \mathrm{g}$ dried sample.

literatures did not have reported on inhibitory activities of $A$. iwayomogi on AR and AGEs formation. For these reasons, we aimed to assess Artemisia iwayomogi, which is native plant in Korea used as usual food, and has potential as anti-diabetic complications agent to inhibit AR and AGEs formation.

Our research led to the isolation of two coumarins (1 and 2), nine flavonoids (3-11), five caffeoylquinic acids (12-16), three diterpene glycosides (17-19), and one phenolic compound (20). Among them, compounds 4, 6, 7, 10, 13, and 18 were first reported on the isolation from A. iwayomogi. Compounds 1-20 have various bioactive properties such as antioxidant, anti-inflammation, antiangiogenesis, and cardiovascular activity [35-37].

After that, the extracts, fractions, and compounds 1-20 from A. iwayomogi were investigated for inhibition of AR. The extracts, $30 \%, 60 \%$, and $100 \% \mathrm{MeOH}$ fractions from $A$. iwayomogi showed significant inhibition of AR more than TMG, positive control. Among compounds isolated from A. iwayomogi, compounds $4,8,9,12,13,14,15$, and 16 were stronger inhibitors than TMG. Out of these, compound 13 exhibited the strongest inhibition. Previous literature reported that caffeic acid had no inhibitory activity on AR but that caffeoyl derivatives are significant inhibitors of AR [24, 38]. Existence of caffeoyl groups for substituents such as quinicacid is important parameter for not only AR inhibitory activity but also various biological activities [39]. Furthermore, other studies have demonstrated relationships between the inhibitory activity and structure of flavonoids [4]. They reported that monohydroxyflavones (such as compound 3) are not inhibitors of AR, that flavonoid glucosides (compounds $\mathbf{8}$ and 9) are more active than flavonoid diglucosides (compounds $\mathbf{1 0}$ and 11), and that flavonoids with a substituted hydroxyl group (compound 6) are more active than those with substituted methoxyl groups (compound 7). Inhibition of AR by flavonoids isolated from A. iwayomogi in our study demonstrated similar results as previous studies. We demonstrated the inhibitory activities of not only AR but also AGEs formation. The $\mathrm{CHCl}_{3}$ fraction from $A$. iwayomogi had no inhibitory activity on AR but showed the best inhibition on AGEs formation. The two coumarins (1 and 2), nine flavonoids (3-11), and five caffeoylquinic acids (12-16) exhibited also great inhibitory activities more than AG, positive control. Among them, compound 1 showed minor inhibition on $\mathrm{AR}$ and exhibited the best inhibition of AGEs formation. Previous literature has reported that the presence of a methoxyl group at C-6 instead of a hydroxyl group and a hydroxyl group at C-7 in the coumarin structure are important parameters in the inhibition of AGEs formation [40]. Therefore, compound 1 may be more active than coumarins with other substituents.

Since A. iwayomogi is found mainly in Korea, we collected 15 samples of $A$. iwayomogi in different regions. The following compounds were analyzed by HPLC-PAD from these fifteen specimens: scopoletin (1), scopolin (2), patulein-3-Oglucoside (8), quercetin-3-gentiobioside (10), rutin (11), 3$O$-caffeoylquinic acid (12), 3,4-di-O-caffeoylquinic acid (14), and 3,5-di-O-caffeoylquinic acid (15). They all showed significant inhibition of either AR or AGEs formation. Overall, the most abundant bioactive compounds were harvested in Yangyang, Gangwon-do, from June (Figure 3). We demonstrated that A. iwayomogi, which was harvested in Yangyang, Gangwon-do, from June, was the best used for medicinal food such as diabetic complications.

\section{Conclusion}

In summary, our study reported the isolation of two coumarins (1 and 2), nine flavonoids (3-11), five caffeoylquinic acids (12-16), three diterpene glycosides (17-19), and one phenolic compound (20). Among them, hispidulin (4), 6-methoxytricin (6), arteanoflavone (7), quercetin-3gentiobioside (10), 1,3-di-O-caffeoylquinic acid (13), and suavioside A (18) were isolated for the first time from $A$. iwayomogi. In addition, two coumarins, nine flavonoids, and five caffeoylquinic acids isolated from A. iwayomogi demonstrated biological activities against AR and AGEs formation. Since extracts of $A$. iwayomogi contained enough amounts of active compounds, $A$. iwayomogi extract can be used for the successful crude herbal drug against AR and AGEs formation. Because A. iwayomogi is native to Korea, we analyzed the bioactive compounds by region. We determined that it is best to harvest A. iwayomogi in Yangyang, Gangwondo, from June in order to get the highest content of active compounds. Based on these, our research demonstrates that $A$. iwayomogi containing active compounds is clearly a potential candidate for a new natural alternative medicine or health supplement and a preventive or therapeutic agent in the treatment of diabetic complications.

\section{Conflicts of Interest}

All authors have no conflicts of interest to declare. 


\section{Acknowledgments}

This research was supported by the Chung-Ang University Graduate Research Scholarship in 2016 and Ministry of Agriculture, Food and Rural Affairs, Republic of Korea.

\section{References}

[1] M. Saraswat, P. Muthenna, P. Suryanarayana, J. M. Petrash, and G. B. Reddy, "Dietary sources of aldose reductase inhibitors: prospects for alleviating diabetic complications," Asia Pacific Journal of Clinical Nutrition, vol. 17, no. 4, pp. 558-565, 2008.

[2] J. G. Collins and C. N. Corder, "Aldose reductase and sorbitol dehydrogenase distribution in substructures of normal and diabetic rat lens," Investigative Ophthalmology \& Visual Science, vol. 16, no. 3, pp. 242-243, 1977.

[3] A. Kato, H. Yasuko, H. Goto, J. Hollinshead, R. J. Nash, and I. Adachi, "Inhibitory effect of rhetsinine isolated from Evodia rutaecarpa on aldose reductase activity," Phytomedicine, vol. 16, no. 2-3, pp. 258-261, 2009.

[4] K. Kawanishi, H. Ueda, and M. Moriyasu, "Aldose reductase inhibitors from the nature," Current Medicinal Chemistry, vol. 10, no. 15, pp. 1353-1374, 2003.

[5] N. Sasaki, R. Fukatsu, K. Tsuzuki et al., "Advanced glycation end products in Alzheimer's disease and other neurodegenerative diseases," American Journal of Pathology, vol. 153, no. 4, pp. 1149-1155, 1998.

[6] H. A. Jung, Y. S. Kim, and J. S. Choi, "Quantitative HPLC analysis of two key flavonoids and inhibitory activities against aldose reductase from different parts of the Korean thistle, Cirsium maackii," Food and Chemical Toxicology, vol. 47, no. 11, pp. 2790-2797, 2009.

[7] S. S. Lim, S. H. Jung, J. Ji, K. H. Shin, and S. R. Keum, "Synthesis of flavonoids and their effects on aldose reductase and sorbitol accumulation in streptozotocin-induced diabetic rat tissues," Journal of Pharmacy and Pharmacology, vol. 53, no. 5, pp. 653668, 2001.

[8] R. L. Kang, O. P. Zee, J. H. Kwak et al., “The polysaccharide fractions of Artemisia species," Korean Journal of Pharmacognosy, vol. 24, pp. 289-295, 1993.

[9] K. A. Koo, J. H. Kwak, K. R. Lee et al., "Antitumor and immunomodulating activities of the polysaccharide fractions from Artemisia selengensis and Artemisia iwayomogi," Archives of Pharmacal Research, vol. 17, no. 5, pp. 371-374, 1994.

[10] S.-H. Lee, Y. Ding, X. T. Yan, Y.-H. Kim, and H.-D. Jang, "Scopoletin and scopolin isolated from Artemisia iwayomogi suppress differentiation of osteoclastic macrophage RAW 264.7 cells by scavenging reactive oxygen species," Journal of Natural Products, vol. 76, no. 4, pp. 615-620, 2013.

[11] S. S. Kim, C. K. Lee, S. S. Kang, H. A. Jung, and J. S. Choi, "Chlorogenic acid, an antioxidant principle from the aerial parts of Artemisia iwayomogi that acts on 1,1-diphenyl-2picrylhydrazyl radical," Archives of Pharmacal Research, vol. 20, no. 2, pp. 148-154, 1997.

[12] Y. Ding, J. A. Kim, S. Y. Yang et al., "Antioxidative sesquiterpenes from Artemisia iwayomogi," Bulletin of the Korean Chemical Society, vol. 32, no. 9, pp. 3493-3496, 2011.

[13] Y. Ding, C. Liang, S. Y. Yang et al., "Phenolic compounds from Artemisia iwayomogi and their effects on osteoblastic MC3T3E1 cells," Biological and Pharmaceutical Bulletin, vol. 33, no. 8, pp. 1448-1453, 2010.
[14] X.-T. Yan, Y. Ding, W. Li et al., "Two new phenolic compounds from Artemisia iwayomogi," Helvetica Chimica Acta, vol. 97, no. 2, pp. 283-289, 2014.

[15] T. S. Park, H. W. Kim, and H. K. Hwang, "Novel use of scopolin and derivatives thereof," Google patents, 2010.

[16] A. R. Kim, Y. N. Zou, T. H. Park et al., "Active components from Artemisia iwayomogi displaying $\mathrm{ONOO}^{-}$scavenging activity," Phytotherapy Research, vol. 18, no. 1, pp. 1-7, 2004.

[17] J.-H. Ryul, H. Ahn, J. Y. Kim, and Y.-K. Kim, "Inhibitory activity of plant extracts on nitric oxide synthesis in LPS-activated macrophages," Phytotherapy Research, vol. 17, no. 5, pp. 485-489, 2003.

[18] J. S. Hwang, H. J. Ji, K. A. Koo et al., "AIP1, a water-soluble fraction from Artemisia iwayomogi, suppresses thymocyte apoptosis in vitro and down-regulates the expression of fas gene," Biological and Pharmaceutical Bulletin, vol. 28, no. 5, pp. 921-924, 2005.

[19] H. J. Ji, H. K. Yeo, N. H. Lee et al., "A carbohydrate fraction, AIP1, from Artemisia iwayomogi down-regulates Fas gene expression and suppresses apoptotic death of the thymocytes induced by 2,3,7,8-tectrachlorodibenzo-p-dioxin," Biotechnology Letters, vol. 27, no. 4, pp. 253-257, 2005.

[20] J.-A. Lee, H.-N. Sung, C.-H. Jeon et al., "A carbohydrate fraction, AIP1 from Artemisia iwayomogi suppresses pulmonary eosinophilia and Th2-type cytokine production in an ovalbumin-induced allergic asthma. Down-regulation of TNF$\alpha$ expression in the lung," International Immunopharmacology, vol. 8, no. 1, pp. 117-125, 2008.

[21] J.-A. Lee, H.-N. Sung, C.-H. Jeon et al., "AIP1, a carbohydrate fraction from Artemisia iwayomogi, modulates the functional differentiation of bone marrow-derived dendritic cells," International Immunopharmacology, vol. 8, no. 4, pp. 534-541, 2008.

[22] S. Sato and P. F. Kador, "Inhibition of aldehyde reductase by aldose reductase inhibitors," Biochemical Pharmacology, vol. 40, no. 5, pp. 1033-1042, 1990.

[23] N. Matsuura, T. Aradate, C. Sasaki et al., "Screening system for the Maillard reaction inhibitor from natural product extracts," Journal of Health Science, vol. 48, no. 6, pp. 520-526, 2002.

[24] H. A. Jung, M. D. N. Islam, Y. S. Kwon et al., "Extraction and identification of three major aldose reductase inhibitors from Artemisia montana," Food and Chemical Toxicology, vol. 49, no. 2, pp. 376-384, 2011.

[25] D. Osei-Safo, M. Chama, I. Addae-Mensah, and R. Waibel, "Hispidulin and other constituents of Scoparia dulcis Linn.", Journal of Science and Technology, vol. 29, no. 2, pp. 7-15, 2009.

[26] V. Martínez, O. Barberá, J. Sánchez-Parareda, and J. Alberto Marco, "Phenolic and acetylenic metabolites from Artemisia assoana," Phytochemistry, vol. 26, no. 9, pp. 2619-2624, 1987.

[27] J. A. Marco, O. Barberá, S. Rodríguez, C. Domingo, and J. Adell, "Flavonoids and other phenolics from Artemisia hispanica," Phytochemistry, vol. 27, no. 10, pp. 3155-3159, 1988.

[28] D. Barron and R. K. Ibrahim, "Ombuin 3-sulphate from Flaveria chloraefolia," Phytochemistry, vol. 27, no. 7, pp. 2362-2363, 1988.

[29] J. D. Su, T. Osawa, S. Kawakishi, and M. Namki, "Antioxidative flavonoids isolated from Osbeckia chinensis L.," Agricultural and Biological Chemistry, vol. 51, no. 10, pp. 2801-2803, 1987.

[30] F. Fathiazad, A. Delazar, R. Amiri, and S. D. Sarker, "Extraction of flavonoids and quantification of rutin from waste Tabacco leaves," Iranian Journal of Pharmaceutical Research, vol. 3, pp. 222-227, 2006. 
[31] N. J. Jun, K. C. Jang, S. C. Kim et al., "Radical scavenging activity and content of cynarin (1,3-dicaffeoylquinic acid) in Artichoke (Cynara scolymus L.)," Journal of Applied Biological Chemistry, vol. 50, pp. 244-248, 2007.

[32] J. Chen, S. Mangelinckx, L. Ma, Z. Wang, W. Li, and N. De Kimpe, "Caffeoylquinic acid derivatives isolated from the aerial parts of Gynura divaricata and their yeast $\alpha$-glucosidase and PTP1B inhibitory activity," Fitoterapia, vol. 99, pp. 1-6, 2014.

[33] Y. Ding, C. Liang, S. Y. Yang, J. H. Kim, Y. M. Lee, and Y. H. Kim, "Iwayoside A, a new diterpene glycoside from Artemisia iwayomogi kitamura that enhances IL-2 secretion," Bulletin of the Korean Chemical Society, vol. 31, no. 8, pp. 2422-2423, 2010.

[34] V. S. Chaturvedula, R. I. San Miguel, and I. Prakash, "Chemical constituents from the polar fraction of Rubus suavissimus," Organic Chemistry: Current Research, vol. 1, article 101, 2011.

[35] Y. Chen, R. Zheng, Z. Jia, and Y. Ju, "Flavonoids as superoxide scavengers and antioxidants," Free Radical Biology and Medicine, vol. 9, no. 1, pp. 19-21, 1990.

[36] R. Pan, Y. Dai, X. Gao, and Y. Xia, "Scopolin isolated from Erycibe obtusifolia Benth stems suppresses adjuvant-induced rat arthritis by inhibiting inflammation and angiogenesis," International Immunopharmacology, vol. 9, no. 7-8, pp. 859-869, 2009.

[37] Y. Sun, X. Ma, and J. Liu, "Compounds from fraction with cardiovascular activity of Chrysanthemum indicum," China Journal of Chinese Materia Medica, vol. 37, no. 1, pp. 61-65, 2012.

[38] Y. S. Lee, Y.-H. Kang, J.-Y. Jung et al., "Inhibitory constituents of aldose reductase in the fruiting body of Phellinus linteus," Biological and Pharmaceutical Bulletin, vol. 31, no. 4, pp. 765768, 2008.

[39] Y. Miyamae, M. Kurisu, J. Han, H. Isoda, and H. Shigemori, "Structure-activity relationship of caffeoylquinic acids on the accelerating activity on ATP production," Chemical and Pharmaceutical Bulletin, vol. 59, no. 4, pp. 502-507, 2011.

[40] H. A. Jung, J. J. Park, M. N. Islam et al., "Inhibitory activity of coumarins from Artemisia capillaris against advanced glycation endproduct formation," Archives of Pharmacal Research, vol. 35, no. 6, pp. 1021-1035, 2012. 


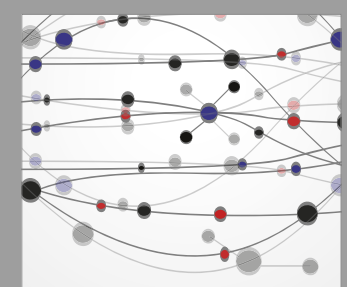

The Scientific World Journal
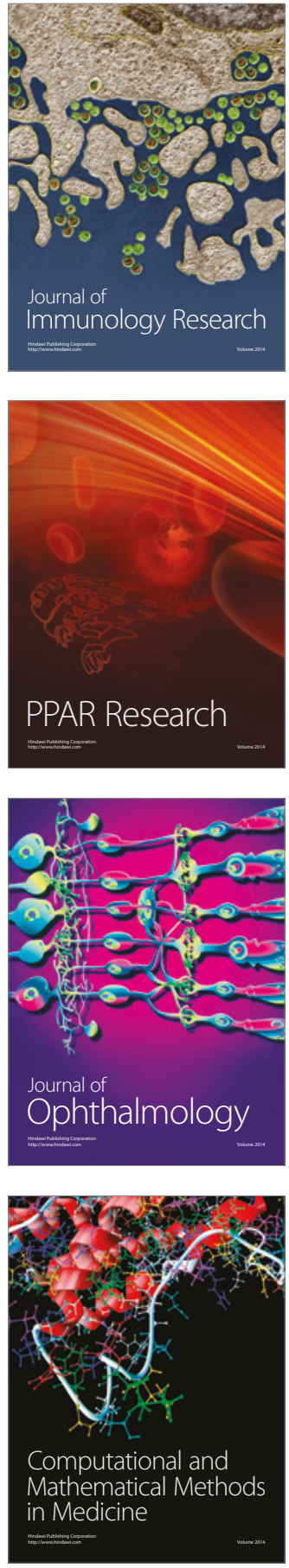

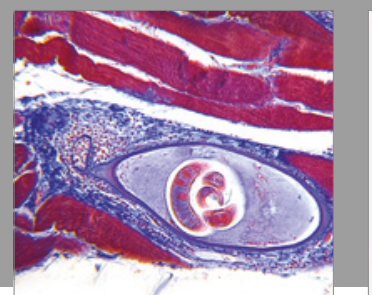

Gastroenterology Research and Practice
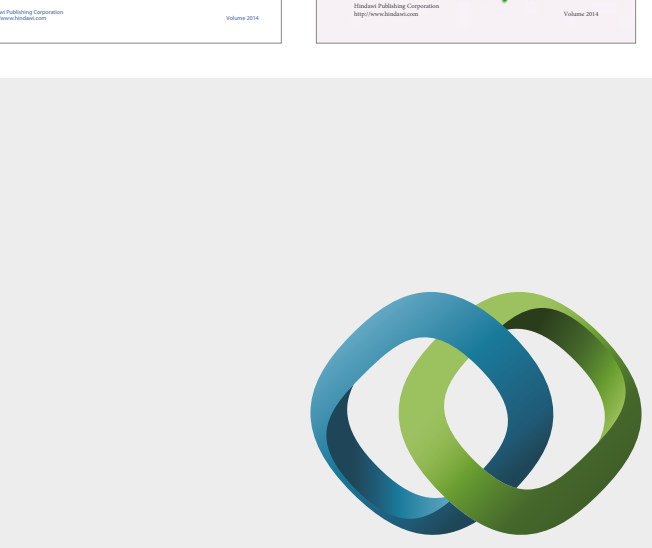

\section{Hindawi}

Submit your manuscripts at

https://www.hindawi.com
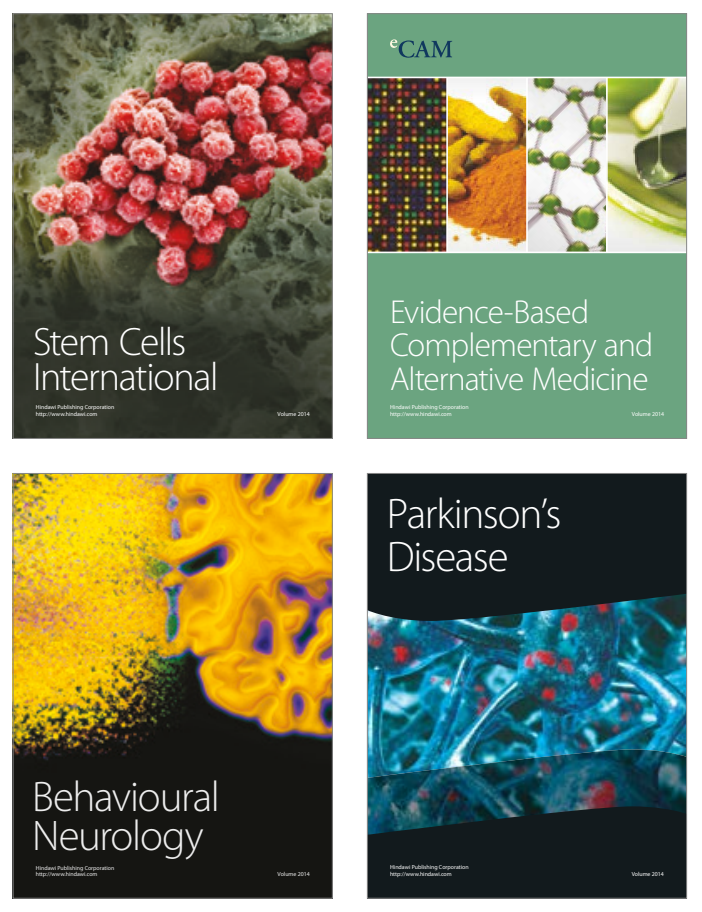
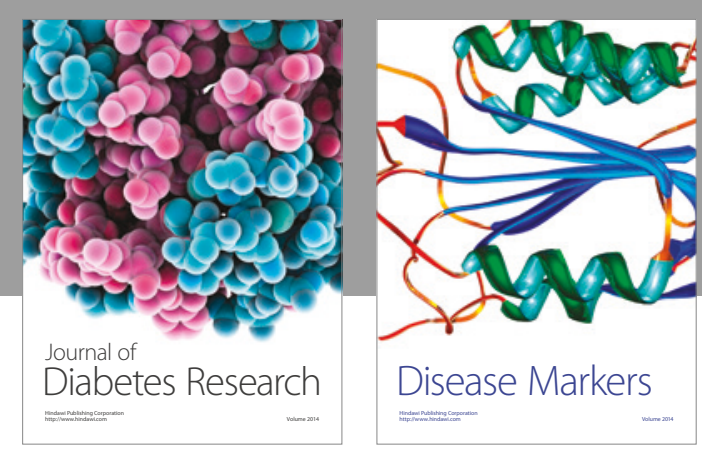

Disease Markers
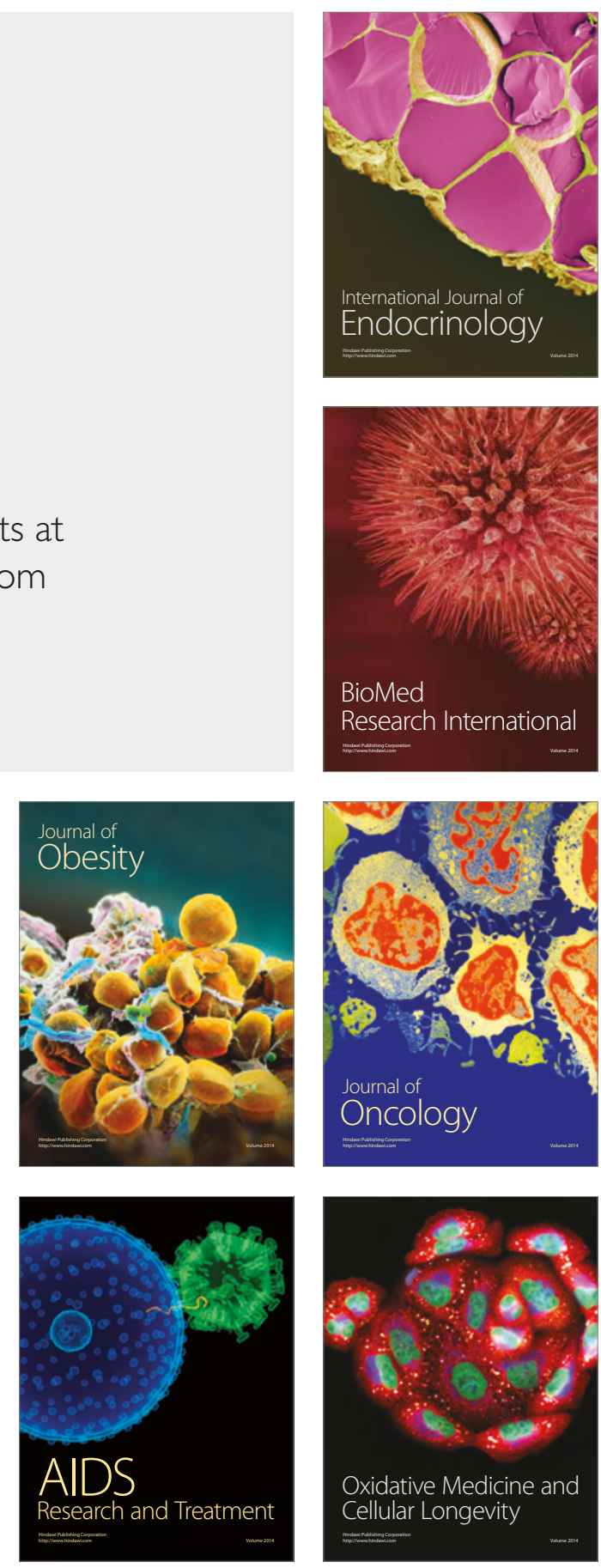